\title{
Voracious vortexes in cataclysmic variables
}

\section{A multi-epoch tomographic study of HT Cassiopeia}

\author{
V. V. Neustroev ${ }^{1}$, S. V. Zharikov², and N. V. Borisov ${ }^{3}$ \\ 1 Astronomy and Space Physics, PO Box 3000, University of Oulu, Oulu 90014, Finland \\ e-mail: vitaly@neustroev.net \\ 2 Instituto de Astronomía, Universidad Nacional Autónoma de México, Apdo. Postal 877, Ensenada, 22800 Baja California, Mexico \\ 3 Special Astrophysical Observatory of the Russian AS, Nizhnij Arkhyz, 369167 Karachaevo-Cherkesia, Russia
}

Received 20 April 2015 / Accepted 4 November 2015

\section{ABSTRACT}

\begin{abstract}
We present multi-epoch, time-resolved optical spectroscopic observations of the dwarf nova HT Cas, which were obtained during 1986, 1992, 1995, and 2005 with the aim of studying the properties of emission structures in the system. We determined that the accretion disc radius, measured from the double-peaked emission-line profiles, is consistently large and lies within the range of $0.45-0.52 a$, where $a$ is the binary separation. This is close to the tidal truncation radius $r_{\max }=0.52 a$. However, this result is not consistent with previous radius measurements. An extensive set of Doppler maps reveals a very complex emission structure of the accretion disc. Apart from a ring of disc emission, the tomograms display at least three areas of enhanced emission: the hot spot from the area of interaction between the gas stream and the disc, which is superposed on the elongated spiral structure, and the extended bright region on the leading side of the disc, which is opposite to the location of the hotspot. The position of the hotspot in all the emission lines is consistent with the trajectory of the gas stream. However, the peaks of emission are located in the range of distances $0.22-0.30 a$, which are much closer to the white dwarf than the disc edge. This suggests that the outer disc regions have a very low density, allowing the gas stream to flow almost freely before it starts to be seen as an emission source. We have found that the extended emission region on the leading side of the disc is always observed at the very edge of the large disc. Observations of other cataclysmic variables, which show a similar emission structure in their tomograms, confirm this conclusion. We propose that the leading side bright region is caused by irradiation of tidally thickened sectors of the outer disc, by the white dwarf and/or hot inner disc regions.
\end{abstract}

Key words. methods: observational - accretion, accretion disks - novae, cataclysmic variables - stars: individual: HT Cas binaries: close

\section{Introduction}

Cataclysmic variables (CVs) are close interacting binary systems that consist of a white dwarf (WD) as primary and a lowmass main-sequence star or a brown dwarf as secondary component (Warner 1995, and references therein). The Roche-lobefilling secondary loses matter via the inner Lagrangian point to the primary. In the absence of a strong magnetic field, the material transferred from the donor star forms an accretion disc around the WD and gradually spirals down onto its surface where it eventually accretes.

Typical optical spectra of CVs are dominated by emission lines of hydrogen and neutral helium series, which are formed in the accretion disc. Lines of other species, such as those from singly ionized helium, calcium, and iron are also often seen (Williams 1983; Honeycutt et al. 1987). The emission lines of CVs with a moderately high orbital inclination are usually very broad with a full-width velocity over several thousand $\mathrm{km} \mathrm{s}^{-1}$ and have a double-peaked profile, which resuls from the Doppler shift of matter that is rotating in a Keplerian disc (Smak 1969, 1981; Horne \& Marsh 1986).

Although the accretion disc is the dominant light source in CVs, many examples show that other emission components may distort the originally symmetric line profile. It is commonly observed that the intensities of the red and blue peaks of the double-peaked profiles are variable with the orbital period phase
(Greenstein \& Kraft 1959). The trailed spectra often show a narrow emission component that moves from one line hump to the other and back during the course of the orbital period, having the form of an "S-wave" (Kraft et al. 1962). This S-wave component is usually attributed to a region of high temperature and luminosity at the outer edge of the accretion disc, which is caused by its interaction with the inflowing gas stream (Smak 1970). This interpretation is supported by the phasing of the S-wave component, which crosses from blue-shifted to redshifted around phase $0.8-0.9$, and which corresponds closely to the expected phasing of this bright area. For the remainder of this paper, we use the slightly outdated term "the hotspot" to refer to the area of interaction between the gas stream and the accretion disc to distinguish the latter from other bright spots on accretion discs.

Soon after, it became apparent that other sources of emission may also be present in an accretion disc. In 1981 Young et al. reported the spectroscopic study of the dwarf nova HT Cas, whose spectra showed unusual behaviour: "The blue wing is stronger near phase 0.0 and the red one stronger at phase 0.5 . This resembles the variations to be expected from an S-wave, but is $180^{\circ}$ out of phase!". The identification of this and other detected emission spots, the phasing of which does not agree with that expected for the hotspot model, was unclear.

The development of Doppler tomography opened up a new regime for the study of accretion structures in interacting 
binaries (Marsh \& Horne 1988). This technique uses the information encoded in spectral line profiles that is taken at different orbital phases to calculate a distribution of emission over the binary. Doppler tomography is now widely used to study interacting binary systems, with the tomograms of dozens of CVs have now been produced. Although a Doppler map is subject to interpretation, since it is created in velocity space, the predicted location of various binary system components in spatial coordinates can easily be translated into velocity coordinates and, hence, compared with the map. Besides the hotspot, this approach helps to identify an irradiated part of secondary stars (Marsh \& Horne 1990), spiral structures in accretion discs (Steeghs et al. 1997), accretion flows in polars (Heerlein et al. 1999), and a "reversed bright spot" caused by the deflected gas stream flow that passes above the disc and hits its back (Neustroev 1998; Neustroev et al. 2011).

Nevertheless, there are observed emission structures that still have no plausible explanation. One of the most mysterious is a bright spot on the leading side of the disc, opposite to the usual location of the hotspot. In trailed spectra, this bright spot produces an S-wave which crosses from blue-shifted to redshifted around phase 0.5. In Doppler images, it is situated in the bottom-right part of the map. This place is far from the region of interaction between the stream and the disc particles. The presence of the leading side bright spot was reported for RR Pic (Schmidtobreick et al. 2003), WX Cet (Tappert et al. 2003), BZ UMa (Neustroev et al. 2006), VW Hyi (Smith et al. 2006), 1RXS J180834.7+101041 (Yakin et al. 2011), V406 Vir (Aviles et al. 2010), EZ Lyn (Zharikov et al. 2013), V2051 Oph (Papadaki et al. 2008; Longa-Peña et al. 2015). Even the tomograms of the famous IP Peg in quiescence occasionally display this kind of bright spot. This list of objects is nowhere near complete. A rough statistical analysis applied to a sample of $68 \mathrm{CVs}$ with published emission-line profile studies showed that the presence of the leading side bright spot is not an exception but a frequent phenomenon (Tappert \& Hanuschik 2001), the source of which has not yet been found.

For these reasons, we were motivated to perform a detailed study of the properties of emission structures in HT Cas, seemingly the first CV where a bright spot in the leading side of the accretion disc has been noticed (Young et al. 1981). In this paper we present and discuss the medium-resolution spectroscopic data obtained during 1986, 1992, 1995, and 2005.

\section{HT Cassiopeiae}

HT Cas was discovered by Hoffmeister (1943) and classified as a U Gem-type star with brightness varying between 13.0 and $16.5 \mathrm{mag}$. The eclipses of HT Cas were discovered by Bond (1978, priv. comm.) and extensively observed by Patterson (1981), who derived an orbital period of $1.77 \mathrm{~h}$ for the system. Borges et al. (2008) report that the orbital period shows period changes of semi-amplitude $\sim 40 \mathrm{~s}$, which seems to repeat on a timescale of about $36 \mathrm{yr}$. The system is characterised by very rare outbursts with mean intervals of 400 days (Wenzel 1987), which suggests an extremely low mass-transfer rate in the system. Moreover, the outbursts occur rather irregularly, e.g. no outbursts were observed from 1989 to 1995 (Kato et al. 2012). In January 1985, Zhang et al. (1986) observed HT Cas in a long and bright outburst and detected superhumps in the light curve of the object, confirming its classification as an SU UMa star. Only two superoutbursts are documented for HT Cas; the only other one after 1985 was observed in 2010 (Kato et al. 2012). The system is also known to exhibit large-amplitude,

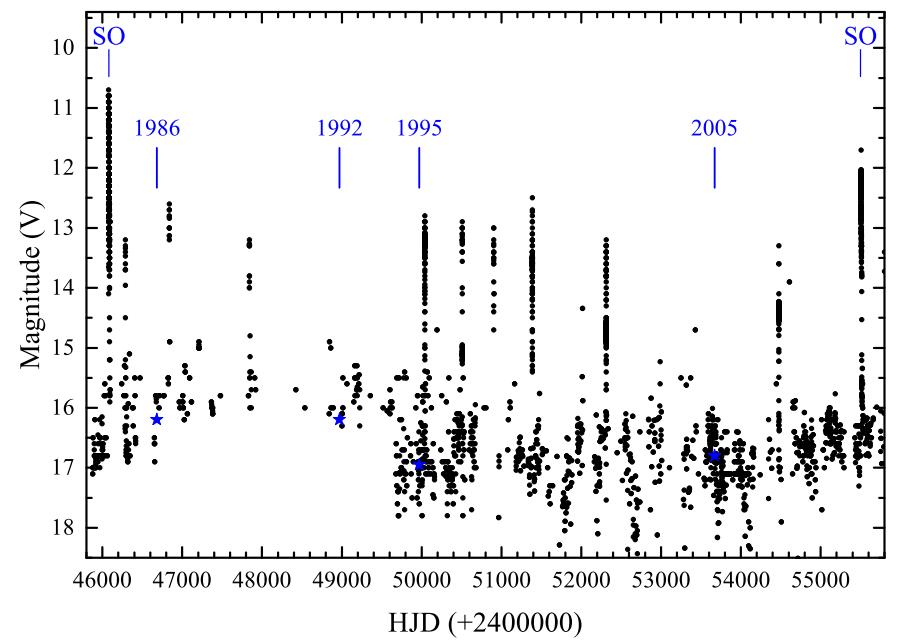

Fig. 1. AAVSO light curve of HT Cas between two superoutbursts in 1985 and 2010, marked by "SO". The blue stars represent averaged magnitudes of the star during our spectroscopic observations.

long-timescale quiescent light variations, from about 15.9 to 17.7 mag (Robertson \& Honeycutt 1996, see also Fig. 1).

Eclipses provide a great opportunity to measure system parameters and to study the accretion disc structure in detail. Patterson (1981) even called HT Cas "the Rosetta Stone of dwarf novae", yet this system might appear to be too atypical for dwarf novae. The light curves and eclipse profiles of HT Cas vary greatly (Patterson 1981; Zhang et al. 1986; Wood et al. 1995). They predominantly show the deep eclipse of the WD, also visible in X-rays (Wood et al. 1995), but the accretion disc component is usually weak and sometimes absent. Orbital humps hotspot modulations - are rarely seen in quiescence. However, the brightness of the hotspot increased significantly during the 2010 superoutburst (Bakkowska \& Olech 2014).

The system parameters for HT Cas are usually taken from Horne et al. (1991): $M_{1}=0.61 \pm 0.04 M_{\odot}, M_{2}=0.09 \pm$ $0.02 M_{\odot}, q=0.15 \pm 0.03, i=81.0 \pm 1.0^{\circ}$. They are based on the derived parameters of a WD eclipse. Several authors studied the accretion disc in HT Cas with the multicolour eclipse-mapping technique. These studies showed that the quiescent disc has a flat brightness temperature profile (5000-7000 K; Wood et al. 1992) and is probably patchy (Vrielmann et al. 2002). Using a similar approach, Feline et al. (2005) reveal changes in the quiescent accretion disc structure, possibly related to variations in the mass-transfer rate from the secondary star. There has been controversy in the literature over the optical thickness of the accretion disc in HT Cas. Zhang et al. (1986) found that the disc is optically thick, Vrielmann et al. (2002) argue that the disc is moderately optically thin, but becomes optically thick near the WD, while Wood et al. (1992) and Feline et al. (2005) claim that the disc is optically thin in both its inner and outer regions.

The optical spectrum of HT Cas is typical for a highinclination dwarf nova, with strong double-peaked emission lines of hydrogen and neutral helium. First spectroscopic observations were made by Rafanelli (1979) who presented a few photographic spectra. Unfortunately, despite a rich history of photometric investigations, HT Cas has been almost neglected by optical high- or medium-resolution spectroscopy. The most recent time-resolved spectroscopic study in the blue wavelength range was presented almost 35 years ago by Young et al. (1981). In particular, they found that the variability of the Balmer emission lines cannot be explained by the canonical hotspot model 
Table 1. Log of spectroscopic observations of HT Cas.

\begin{tabular}{ccccccc}
\hline \hline Set & Date & $\begin{array}{c}\text { Telescope/ } \\
\text { instrument }\end{array}$ & $\begin{array}{c}\lambda \text { range } \\
(\AA)\end{array}$ & $\begin{array}{c}\text { Exp. time } \\
(\mathrm{s})\end{array}$ & $\begin{array}{c}\text { Number } \\
\text { of exps. }\end{array}$ & $\begin{array}{c}\text { Duration } \\
(\mathrm{h})\end{array}$ \\
\hline Set-1986 & 1986-Sep.-08 & $6.0 \mathrm{~m}$ / SP-124 & $3600-5500$ & 300 & 15 & 1.77 \\
& 1986-Sep.-09 & $6.0 \mathrm{~m} / \mathrm{SP}-124$ & $5400-7300$ & 360 & 16 & 2.12 \\
Set-1992 & 1992-Dec.-16 & $6.0 \mathrm{~m}$ / SP-124 & $3600-5500$ & 300 & 14 & 1.41 \\
Set-1995 & $1995-S e p .-09$ & $6.0 \mathrm{~m} / \mathrm{SP}-124$ & $3860-5800$ & 180 & 58 & 3.54 \\
Set-2005 & 2005-Oct.-29 & $2.1 \mathrm{~m} / \mathrm{B} \& \mathrm{Ch}$ & $4600-6700$ & 293 & 40 & 3.54 \\
& 2005-Oct.-31 & $2.1 \mathrm{~m} / \mathrm{B} \& \mathrm{Ch}$ & $6150-7225$ & 293 & 40 & 3.54 \\
\hline
\end{tabular}

and that the semi-amplitude $K_{1}$ of their radial-velocity variations is about $115 \mathrm{~km} \mathrm{~s}^{-1}$. Yet the radial velocity curves are shifted by $30^{\circ}$ relative to the eclipse. Horne et al. (1991) concluded in their work that the $K_{1}$ velocity of $115 \mathrm{~km} \mathrm{~s}^{-1}$ is unreliable and predicted $K_{1}$ to be $58 \pm 11 \mathrm{~km} \mathrm{~s}^{-1}$. This has been neither confirmed nor denied until now. There was one more spectroscopic study of HT Cas performed by Marsh (1990) with the low-resolution data. Marsh was able to detect absorption lines from the M5.4 \pm 0.3 secondary star and to measure its radial velocity semiamplitude $K_{2}=430 \pm 25 \mathrm{~km} \mathrm{~s}^{-1}$. However, no attempt was made to study the emission lines in detail and to derive $K_{1}$.

Among other peculiar characteristics of HT Cas, there is an unusually small radius of the accretion disc $R_{\mathrm{d}}$ as inferred by many researchers (Zhang et al. 1986; Horne et al. 1991; Vrielmann et al. 2002; Feline et al. 2005). A typical value of $R_{\mathrm{d}}$ in quiescence was measured as being $\sim 0.23 a$. This is only a bit larger than the circularization radius $(0.195 a$, Verbunt $\&$ Rappaport 1988), which determines the theoretically allowed minimal accretion disc radius. We note that most measurements of $R_{\mathrm{d}}$ were based on the position of the hotspot. In Sect. 5.1 we show that the position of the hotspot does not always give reliable estimates for the accretion disc radius.

\section{Observations and data reduction}

The spectra presented here were obtained during four observing runs in 1986, 1992, 1995, and 2005. To check the photometric state of HT Cas during each set of spectroscopic observations, we also obtained several photometric measurements on accompanying telescopes. These observations indicate that, although HT Cas has always remained in quiescence, its brightness changed substantially. During the observations in 1986 and 1992, the $V$ magnitude was about $16.2 \mathrm{mag}$, whereas in 1995 and 2005 it was $\sim 17.0$ and $\sim 16.8 \mathrm{mag}$, respectively (Fig. 1).

The first three observations were conducted in 1986, 1992, and 1995 at the Special Astrophysical Observatory of the Russian Academy of Sciences, using a 1024-channel television scanner mounted on the SP-124 spectrograph at the Nasmyth-1 focus of the 6-m telescope (Drabek et al. 1986). The observations in 1986 were taken during two consecutive nights of September 8 and 9, about 20 months after the superoutburst. The spectra were taken in the wavelength ranges of 3600-5210 (blue spectra) and 5410-7260 $\AA$ (red spectra) respectively with a dispersion of $1.9 \AA$ channel $^{-1}$. The corresponding spectral resolution was about $4.5 \AA$. A total of 15 blue and 16 red spectra were obtained with 300 and $360 \mathrm{~s}$ individual exposures. About 1.0 and 1.25 orbital cycles were covered each night. The observations on December 16, 1992 and September 9, 1995 were conducted with the same dispersion in only the blue wavelength range (4000-5020 $\AA$ and 3900-5780 , respectively). 14 and 58 spectra, with 300 and 180 s individual exposures, were taken during these runs, which covers about 0.8 and 2.0 orbital cycles. We note that the 1995 observations were performed about two months before the normal outburst, which was particularly well observed by Ioannou et al. (1999). Because of electronic focus issues with the TV scanner during the 1995 observations, the spectral resolution of these data degrades rather suddenly toward longer wavelengths, especially beyond $\sim 4500 \AA$. However, the quality of the spectra in shorter wavelengths is still reasonably good and suitable for analysis. Comparison spectra of a $\mathrm{HeNeAr}$ lamp were used for the wavelength calibration. No attempt at flux calibration was made for these data. The data reduction of the SAO observations was carried out using the procedure described by Kniazev (1994). Hereafter we will refer to these data sets as set-1986, set-1992, and set-1995.

Further observations were obtained in 2005 during two nights of October 29 and 31 at the Observatorio Astronomico Nacional (OAN SPM) in Mexico on the 2.1-m telescope with the Boller \& Chivens spectrograph, which was equipped with a $24 \mu \mathrm{m}(1024 \times 1024)$ SITe CCD chip. The observations on the first night were taken in the wavelength range of 4600-6700 with a dispersion of $2.05 \AA$ pixel $^{-1}$ while the rest of the spectra were obtained in the wavelength range of 6150-7225 $\AA$ with a dispersion of $1.05 \AA$ pixel $^{-1}$. The corresponding spectral resolution was about 4.1 and $2.2 \AA$, respectively. A total of 40 spectra with $293 \mathrm{~s}$ individual exposures were taken each night, covering exactly two orbital periods. Comparison spectra of a $\mathrm{CuHeNeAr}$ lamp taken during the night were used for the wavelength calibration. Both nights of observations were photometric with the seeing ranged from 1 to 2 arcsec. To apply an accurate flux correction, two standard spectrophotometric stars at different airmasses were observed every night. They were selected from Feige110, HR3454, and G191-B2B (Oke 1990). The data reduction was performed using the IRAF environment. We will refer to these data as set-2005, sometimes dividing it into set-2005-n1 and set-2005-n 2 for the first and second nights of observations, if necessary. A log of observations is presented in Table 1.

The orbital phases of the spectra were calculated using the linear ephemeris of Feline et al. (2005). The uncertainties of this ephemeris in the orbital phase at the time of our observations are negligible. For the 2005 data, for which the propagated error is larger, it is less than $2 \times 10^{-4}$.

\section{Data analysis and results}

\subsection{Averaged spectra and their long-term variability}

The averaged and continuum-normalised out-of-eclipse spectra of HT Cas, uncorrected for orbital motion, are shown in Fig. 2. Here and elsewhere in this paper, out-of-eclipse phases are defined as $0.1 \leq \varphi \leq 0.9$. The averaged spectra are similar in appearance to those presented by Young et al. (1981) and Williams (1983). They are dominated by extremely strong and 


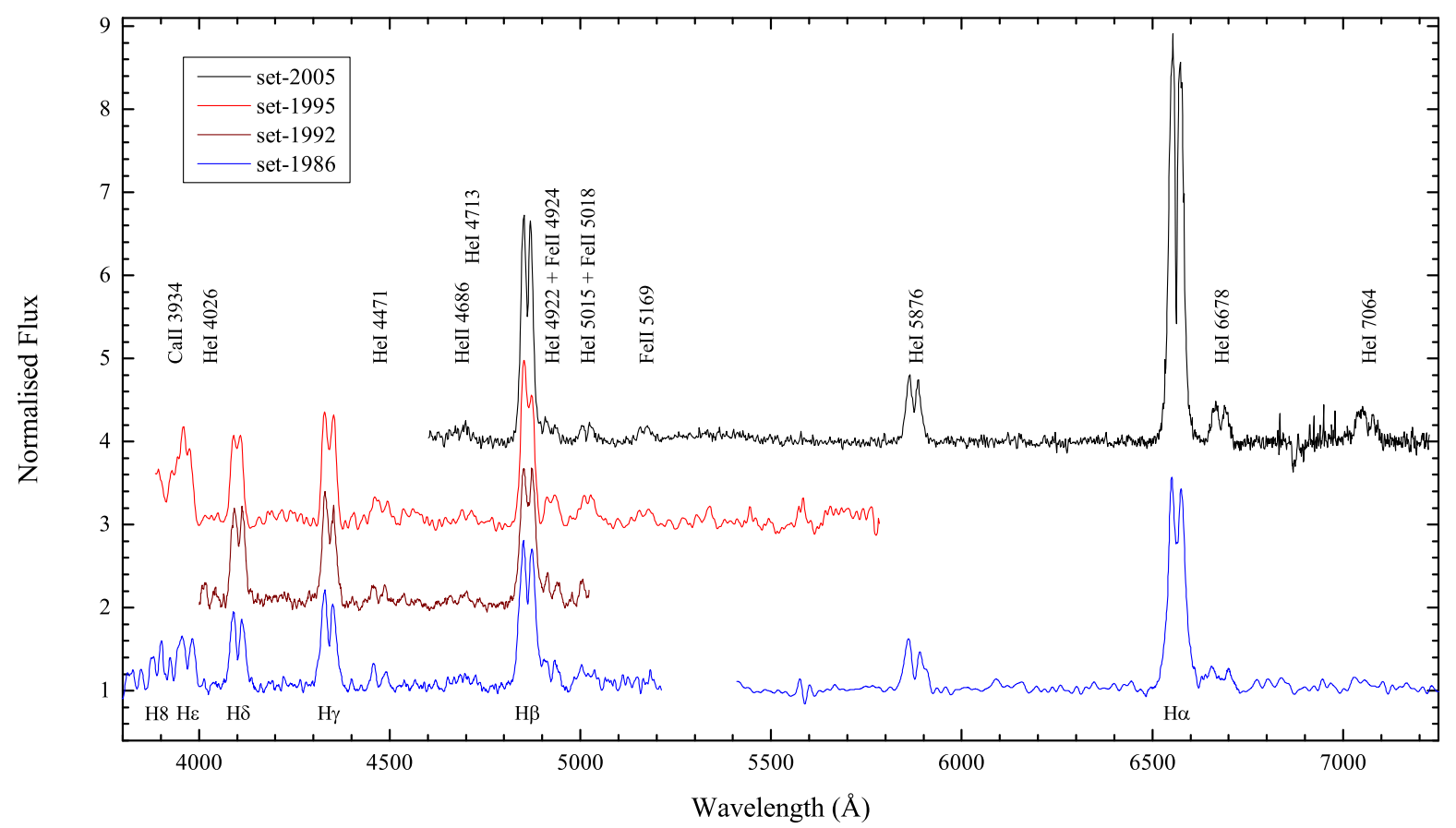

Fig. 2. Combined and continuum-normalised out-of-eclipse spectra of HT Cas for each epoch of observation. Spectra shifted vertically to prevent overlap.

broad double-peaked emission lines of the Balmer series. Apart from hydrogen, numerous weaker emission lines of neutral helium and singly ionized iron (Fe II) are present. Also, the high excitation line of He II $\lambda 4686$ is clearly detected. Table 2 outlines different parameters of the most prominent lines that were measured from the averaged spectra. In Tables 3 and 4, we also separately present the equivalent width (EW) measurements, including those available in the literature, and the corresponding Balmer and neutral helium decrement values, respectively.

A comparison of the averaged spectra from different data sets shows significant quantitative differences between them. There are notable variations in both emission-line strengths and their ratios for different lines. For example, the $\mathrm{EW}$ of $\mathrm{H} \alpha$ in the set1986 is $\sim 126 \AA$, but in the set-2005 it reaches almost $200 \AA$. The Balmer decrement, being relatively flat in the sets 1992 and 1995, appears rather steep in the set-1986 and especially in the set-2005, indicating optically thin conditions in the outer parts of the accretion disc. The decrement within various series of neutral helium lines (e.g., the singlets $\lambda 6678 / \lambda 4922$ and triplets $\lambda 5876 / \lambda 4471)$, the relative strengths of the He I triplet and singlet lines (e.g., $\lambda 5876 / \lambda 6678$ ) and the ratio of hydrogen to He I strengths have also changed substantially (Table 4). This implies that the opacity and the optical thickness of the disc have varied over time, but these variations do not seem to correlate directly with the system flux (see the last column in Table 3).

\subsection{Light and colour curves}

The spectra from the set-2005 were used to construct light curves. The blue and red continuum light curves were computed by summing the flux in the wavelength ranges $\lambda \lambda 4740-4800$ and $\lambda \lambda 6400-6500 \AA$, respectively. The emission-line light curves for $\mathrm{H} \alpha, \mathrm{H} \beta, \mathrm{He}$ I $\lambda 5876$, and He II $\lambda 4686$ were computed by summing the continuum-subtracted flux inside of $\pm 2700 \mathrm{~km} \mathrm{~s}^{-1}$ window that was centred at the emission-line wavelengths. The resulting light curves are plotted in Fig. 3 (left-hand panel).
Although an insufficient phase resolution of the data does not allow us to investigate the eclipse profiles in detail, we are still able to see their most distinctive features. The continuum shows a deep almost symmetrical eclipse, with the blue light curve having a deeper eclipse than the red. There is a weak sign of an orbital hump around phase $0.8-0.9$, which is more easily seen in the blue light, consistent with the presence of the hotspot. The orbital hump is stronger in the Balmer and He I lines. The eclipses of the emission lines have a different shape from the continuum, exhibiting a distinctive shoulder during egress. The latter feature is seen in both the Balmer, He I, and He II lines, except for the $\mathrm{H} \alpha$ line from the set-2005-n2. We note that even though the continuum flux was nearly the same during two nights of the 2005 observations, the $\mathrm{H} \alpha$ line appeared to be slightly stronger on the second night.

Figure 3 (right-hand panel) also shows the flux ratios of the continuum segments and Balmer and He I emission lines. The ratio of the blue and red continuum fluxes (an equivalent of a colour index) shows little variation outside of eclipses, but there is a sign of an orbital hump with maximum at phase $\sim 0.95$, during which the continuum appears bluer. That is consistent with the presence of the hotspot. The binary becomes much redder in the middle of the eclipse. The $\mathrm{H} \alpha / \mathrm{H} \beta$ and $\mathrm{He} \mathrm{I} \lambda 5876 / \mathrm{H} \beta$ ratios show synchronous sinusoidal variations, with the lowest value also observed at phase $0.95-1.0$. The $\mathrm{H} \alpha / \mathrm{H} \beta$ ratio varies by $\sim 10$ percent around a mean value and there is a notable zigzag jump in the first half of the eclipse. The $\mathrm{He} \mathrm{I} \lambda 5876 / \mathrm{H} \beta$ ratio varies considerably by a factor of $\sim 2$ more than the $\mathrm{H} \alpha / \mathrm{H} \beta$. However, the data show no clear evidence for a jump during the eclipse.

\subsection{Accretion disc parameters from modelling of the emission-line profiles}

All emission lines exhibit, in the averaged spectra, slightly asymmetric double-peaked profiles, with a blue peak being stronger 
V. V. Neustroev et al.: Multi-epoch tomographic study of HT Cas

Table 2. Parameters of the most prominent emission lines in the averaged spectra of HT Cas.

\begin{tabular}{|c|c|c|c|c|c|c|c|c|}
\hline \multirow[t]{2}{*}{ Set } & \multirow{2}{*}{$\begin{array}{l}\text { Spectral } \\
\text { line }\end{array}$} & \multirow{2}{*}{$\begin{array}{c}\text { Flux } \\
\left(\mathrm{erg} \mathrm{s}^{-1} \mathrm{~cm}^{-2}\right)\end{array}$} & \multirow{2}{*}{$\begin{array}{l}\text { Relative } \\
\text { intensity }\end{array}$} & \multirow{2}{*}{$\begin{array}{l}F W H M \\
\left(\mathrm{~km} \mathrm{~s}^{-1}\right)\end{array}$} & \multirow{2}{*}{$\begin{array}{l}\text { Peak-to-peak } \\
\left(\mathrm{km} \mathrm{s}^{-1}\right)\end{array}$} & \multicolumn{3}{|c|}{ Model parameters } \\
\hline & & & & & & $V_{\text {out }}\left(\mathrm{km} \mathrm{s}^{-1}\right)$ & $b$ & $r_{\text {in }} / r_{\text {out }}$ \\
\hline \multirow[t]{10}{*}{1986} & $\mathrm{H} \alpha$ & & 3.50 & 2130 & 1120 & $590 \pm 5$ & $1.59 \pm 0.03$ & $0.06 \pm 0.01$ \\
\hline & $\mathrm{H} \beta$ & & 2.75 & 2865 & 1370 & $620 \pm 21$ & $2.03 \pm 0.08$ & $0.07 \pm 0.01$ \\
\hline & $\mathrm{H} \gamma$ & & 2.10 & 3030 & 1450 & & & \\
\hline & $\mathrm{H} \delta$ & & 1.89 & 3200 & 1750 & & & \\
\hline & He I $\lambda 4471$ & & 1.28 & 3150 & 2090 & & & \\
\hline & He I $\lambda 4922$ & & 1.36 & & 1850 & & & \\
\hline & He I $\lambda 5015$ & & 1.28 & 3500 & 2030 & & & \\
\hline & Fe II $\lambda 5169$ & & 1.20 & & & & & \\
\hline & He I $\lambda 5876$ & & 1.50 & 2860 & 1500 & $690 \pm 9$ & $2.36 \pm 0.03$ & $0.08 \pm 0.01$ \\
\hline & He I $\lambda 6678$ & & 1.40 & 2150 & 1080 & & & \\
\hline \multirow[t]{5}{*}{1992} & $\mathrm{H} \beta$ & & 2.68 & 2720 & 1360 & $742 \pm 8$ & $1.42 \pm 0.08$ & $0.06 \pm 0.01$ \\
\hline & $\mathrm{H} \gamma$ & & 2.30 & 2800 & 1570 & & & \\
\hline & $\mathrm{H} \delta$ & & 2.20 & 2990 & 1520 & & & \\
\hline & He I $\lambda 4471$ & & 1.27 & & 2010 & & & \\
\hline & Не I $\lambda 4922$ & & 1.34 & & 1700 & & & \\
\hline \multirow[t]{7}{*}{1995} & $\mathrm{H} \beta$ & & 2.75 & 2440 & 1250 & & & \\
\hline & $\mathrm{H} \gamma$ & & 2.32 & 2830 & 1670 & & & \\
\hline & $\mathrm{H} \delta$ & & 2.08 & 2670 & 1264 & & & \\
\hline & Не І $\lambda 4471$ & & 1.29 & & & & & \\
\hline & Не I $\lambda 4922$ & & 1.34 & & & & & \\
\hline & He I $\lambda 5015$ & & 1.36 & & & & & \\
\hline & Fe II $\lambda 5169$ & & 1.18 & & & & & \\
\hline \multirow[t]{7}{*}{ 2005-n1 } & $\mathrm{H} \alpha$ & $8.7 \times 10^{-14}$ & 5.55 & 1960 & 1070 & $572 \pm 6$ & $0.81 \pm 0.04$ & $0.04 \pm 0.01$ \\
\hline & $\mathrm{H} \beta$ & $7.4 \times 10^{-14}$ & 3.70 & 2160 & 1090 & $571 \pm 11$ & $1.58 \pm 0.08$ & $0.09 \pm 0.01$ \\
\hline & He I $\lambda 4922$ & $6.6 \times 10^{-15}$ & 1.21 & & 1520 & & & \\
\hline & He I $\lambda 5015$ & $6.9 \times 10^{-15}$ & 1.20 & 2340 & 1260 & & & \\
\hline & Fe II $\lambda 5169$ & $5.0 \times 10^{-15}$ & 1.19 & & 1120 & & & \\
\hline & He I $\lambda 5876$ & $2.1 \times 10^{-14}$ & 1.77 & 2130 & 1160 & $606 \pm 7$ & $1.41 \pm 0.04$ & $0.02 \pm 0.01$ \\
\hline & He II $\lambda 4686$ & $4.7 \times 10^{-15}$ & 1.15 & & 1640 & & & \\
\hline \multirow[t]{3}{*}{ 2005-n2 } & $\mathrm{H} \alpha$ & $1.03 \times 10^{-13}$ & 5.65 & 1960 & 920 & $581 \pm 8$ & $0.95 \pm 0.09$ & $0.06 \pm 0.02$ \\
\hline & He I $\lambda 6678$ & $1.0 \times 10^{-14}$ & 1.42 & 2150 & 1080 & & & \\
\hline & He I $\lambda 7064$ & $8.8 \times 10^{-15}$ & 1.35 & & 1140 & & & \\
\hline
\end{tabular}

Table 3. Equivalent widths of HT Cas.

\begin{tabular}{lccccccccccccc}
\hline \hline Set & Ref. & $\mathrm{H} \alpha$ & $\mathrm{H} \beta$ & $\mathrm{H} \gamma$ & $\mathrm{H} \delta$ & $\lambda 4471$ & $\lambda 4922$ & $\lambda 5015$ & $\lambda 5169$ & $\lambda 5876$ & $\lambda 6678$ & $\lambda 7064$ & Magnitude $(V)$ \\
\hline 1980 & 1 & - & 77.4 & 51.0 & 41.1 & 11.7 & 9.2 & 9.5 & - & - & - & - & 16.0 \\
1981 & 2 & 233.0 & 98.0 & 72.8 & 61.7 & 16.0 & 11.0 & 12.0 & 12.0 & 32.0 & 32.0 & - & 16.2 \\
1986 & 3 & 125.9 & 82.0 & 47.9 & 37.9 & 10.2 & 15.0 & 14.3 & 7.2 & 27.4 & 17.4 & - & 16.2 \\
1988 & 4 & 186.4 & - & - & - & - & - & - & - & 34.1 & 17.4 & 16.1 & 16.2 \\
1992 & 3 & - & 76.1 & 54.3 & 48.4 & 12.0 & 12.6 & - & - & - & - & - & 16.2 \\
1995 & 3 & - & 69.9 & 50.7 & 39.3 & 14.5 & 13.3 & 16.5 & 8.0 & - & - & - & 17.0 \\
2005 & 3 & 198.5 & 98.0 & - & - & - & 7.5 & 7.9 & 6.2 & 30.4 & 19.7 & 18.2 & 16.8 \\
\hline
\end{tabular}

References. (1) Young et al. (1981): the spectra were taken on 1980 August 1; (2) Williams (1983): the spectra were taken on 1981 December 24-27; (3) This paper; (4) Marsh (1990): the spectra were taken on 1988 July 22-24.

than the red one. The lines are very broad with a full width at zero intensity (FWZI) of up to 5-6 thousand $\mathrm{km} \mathrm{s}^{-1}$ and a peak-to-peak separation of $\gtrsim 1100 \mathrm{~km} \mathrm{~s}^{-1}$ (in $\mathrm{H} \alpha$ ), which increases monotonically towards the higher order Balmer lines. These properties suggest the origin of emission lines in an accretion disc (Smak 1981; Horne \& Marsh 1986). A comparison of the emission lines from different data sets shows that they vary not only in relative intensity but also in shape and peak-to-peak separation of the profiles. Figure 4 shows the $\mathrm{H} \alpha$ and $\mathrm{H} \beta$ profiles which exhibit notably different slopes of the line wings.

It is well known that the velocity-separation between peaks in the double-peaked profiles is defined by the velocity of the outer rim of the accretion disc $V_{\text {out }}$ which, in turn, depends on its radius (Smak 1981). The shape of the line wings is controlled by the surface radial emissivity profile (Smak 1981; Horne \& Marsh 1986), which is commonly assumed to follow a power-law function of the form $f(r) \propto r^{-b}$, where $r$ is the radial distance from the WD. To estimate accretion disc parameters, we fitted the averaged emission line profiles using a simple model of a uniform flat axisymmetric Keplerian geometrically thin disc (Smak 1981; Horne \& Marsh 1986). The primary free parameters of the model are:

1. $V_{\text {out }}$, the velocity of the outer rim of the accretion disc;

2. $b$, the power-law index of the line emissivity profile $f(r)$;

3. $r_{\text {in }} / r_{\text {out }}$, the ratio of the inner to the outer radii of the disc.

Examples of the application of this technique to the real data are given in Neustroev (1998), Neustroev et al. (2002, 2014). 
Table 4. Balmer and He I decrements.

\begin{tabular}{ccccccccc}
\hline \hline Set & Ref. & $\mathrm{H} \alpha / \mathrm{H} \beta$ & $\mathrm{H} \gamma / \mathrm{H} \beta$ & $\mathrm{H} \delta / \mathrm{H} \beta$ & $\mathrm{He}$ I $\lambda 6678 / \lambda 4922$ & $\mathrm{He}$ I $\lambda 5876 / \lambda 4471$ & $\mathrm{He}$ I $\lambda 5876 / \lambda 6678$ & $\mathrm{H} \beta / \mathrm{He}$ I $\lambda 4922$ \\
\hline 1980 & 1 & - & 0.66 & 0.53 & - & - & - & 8.37 \\
1981 & 2 & 2.38 & 0.74 & 0.63 & 2.91 & 2.00 & 1.00 & 8.91 \\
1986 & 3 & 1.54 & 0.58 & 0.46 & 1.16 & - & 1.57 & 5.47 \\
1988 & 4 & - & - & - & - & - & - & - \\
1992 & 3 & - & 0.71 & 0.64 & - & - & - & 5.03 \\
1995 & 3 & - & 0.72 & 0.56 & - & - & 1.54 & 13.26 \\
2005 & 3 & 2.03 & - & - & 2.63 & & & - \\
\hline
\end{tabular}

References. (1) Young et al. (1981); (2) Williams (1983); (3) This paper; (4) Marsh (1990).
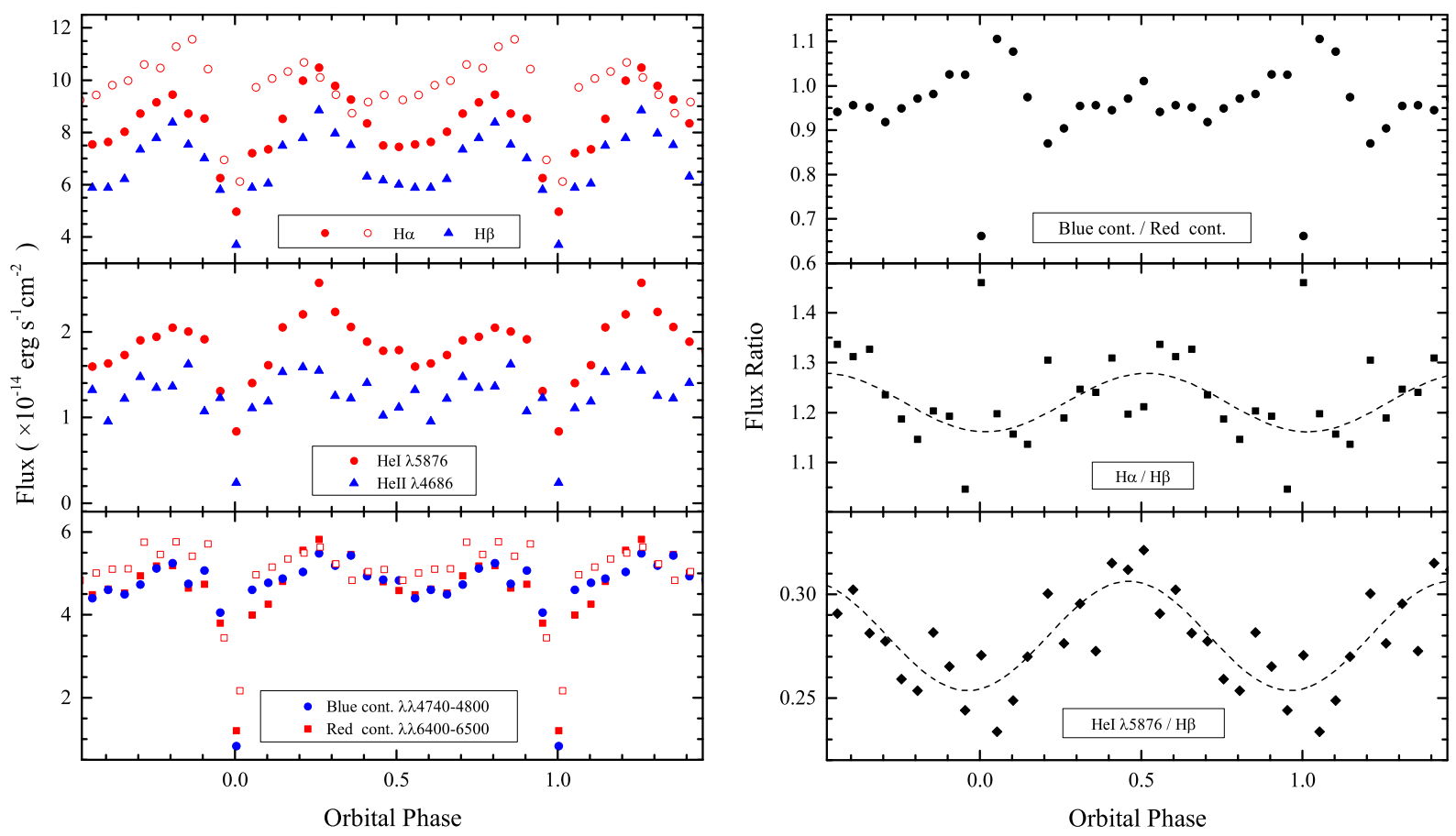

Fig. 3. Left: continuum and emission-line light curves. The filled symbols represent the data from the set-2005-n1, the open symbols are for the set-2005-n2. Right: continuum and emission-line flux ratios.

The best-fitting model parameters for the major emission lines are listed in Table 2 and the errors were estimated with a Monte Carlo approach described in Borisov \& Neustroev (1997). The model fits for the $\mathrm{H} \alpha$ and $\mathrm{H} \beta$ emission-line profiles from the 1986, 1992, and 2005 data sets are shown in Fig. 4 by dashed lines.

Observations of CVs show that the power-law index $b$ is usually in the range of $1-2$, rarely being less than 1.5 (Horne \& Saar 1991 ). Most of our model fits also give $b \approx 1.5-2.0$. However, the best-fitting index $b$ for $\mathrm{H} \alpha$ from the set-2005 is $<1.0$. This suggests a flatter radial distribution of the emission-line flux from the accretion disc of HT Cas during the 2005 observations. Marsh \& Horne (1990) argued that, to explain such a behaviour, an increased role of photoionization by the soft X-rays and UV photons from the centre of the accretion disc should be taken into account. Coupled with the significantly steeper Balmer and He I decrements in the 2005 spectra, this may also suggest a lowering of gas density in an outer area of the disc producing $\mathrm{H} \alpha$ emission.

\subsection{Emission-line variations and Doppler tomography}

Young et al. (1981) mentioned that the emission lines of HT Cas do not vary much in profile around the orbit (see Fig. 1 in their paper). Our observations confirm this finding as well as the most mysterious property of profile variations: the blue peak of lines is stronger at phase $0.1-0.2$ and the red peak is stronger near phase $\sim 0.6$, which is $180^{\circ}$ out of phase than is expected from the ordinary S-wave (Fig. 5). However, this is correct for the $\mathrm{H} \alpha$ and $\mathrm{H} \beta$ lines only, whereas the $\mathrm{He}$ I lines show the opposite, usual behaviour.

More details are revealed in the trailed spectra. In top and bottom panels of Fig. 6, the most representative lines from the data sets 2005-n1 and 2005-n2 are shown. One can clearly recognize that the ordinary S-wave is certainly present in most of the lines, yet the anomalous emission source mentioned above is strong in $\mathrm{H} \alpha$, weaker in $\mathrm{H} \beta$, and very weak or undetectable in $\mathrm{He}$ I lines. We note that even though the He II $\lambda 4686$ line is clearly seen in averaged spectra, it is still too weak and noisy to reveal any variability.

To provide a more convincing picture of the sources of emission in the accretion disc of HT Cas, we used Doppler tomography (Marsh \& Horne 1988). For a comprehensive review of the method and many examples of its application, see Marsh (2001) and references therein. Figures 6 and 7 show the Doppler maps computed using the code developed by Spruit (1998).

We start the discussion with the 2005 data set as these spectra have higher signal-to-noise ratio $(\mathrm{S} / \mathrm{N})$ and spectral resolution 


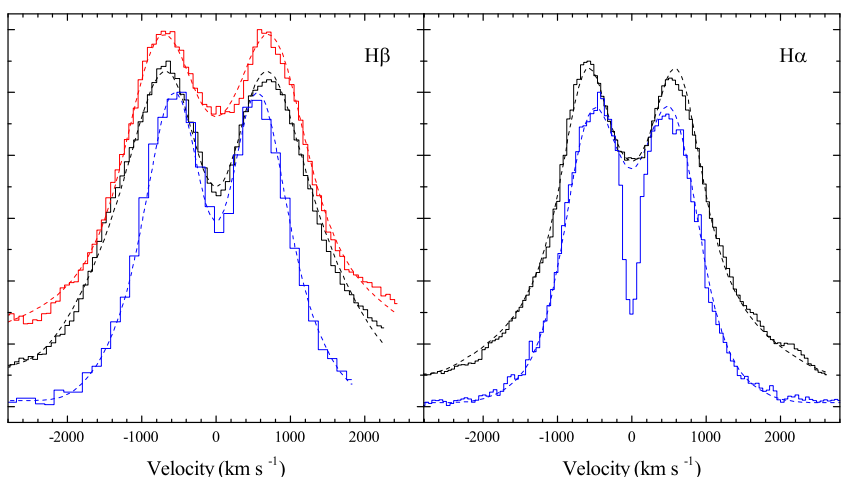

Fig. 4. Averaged profiles of the $\mathrm{H} \alpha$ and $\mathrm{H} \beta$ emission lines, observed in 1992, 1986, and 2005 (red, black, and blue lines, respectively) together with the corresponding model fits (dashed lines). The 1992, 1986 , and 2005 profiles are shifted vertically by $10 \%$ to prevent overlap.

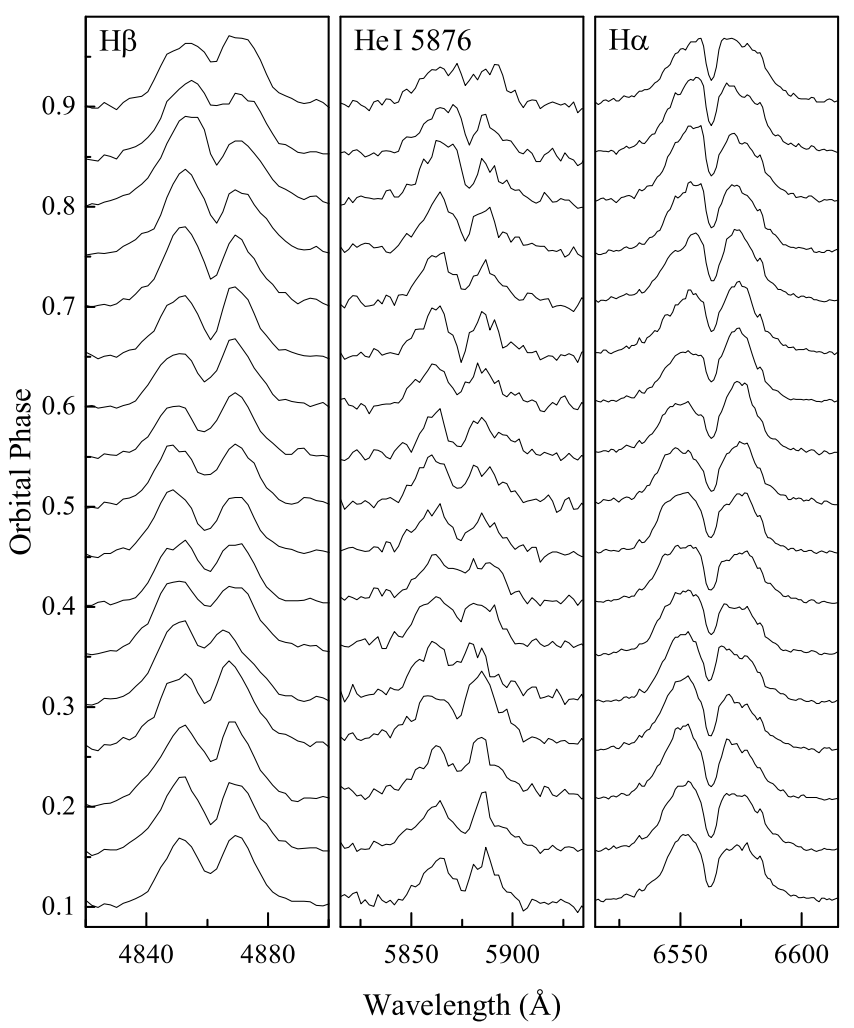

Fig. 5. Evolution of the out-of-eclipse emission-line profiles during the set-2005-n1 (H $\beta$ and He I 5876) and the set-2005-n2 (H $\alpha)$. The spectra have been ordered according to phase.

and they produce maps of the best quality. The tomograms of the representative lines from this set are shown in Fig. 6, together with the corresponding reconstructed counterparts, shown in the top and bottom panels, alongside the trailed spectra. Since the gradual occultation of the emitting regions during eclipse is not taken into account, we constrained our data sets by removing eclipse spectra covering the phase ranges $\phi=0.9-1.1$. To help in interpreting the Doppler maps, the positions of the WD, the centre of mass of the binary and the Roche lobe of the secondary star are marked. The predicted trajectory of the gas stream and the Keplerian velocity of the disc along the gas stream have also been shown in the form of the lower and upper curves, respectively. The Roche lobe of the secondary and the trajectories were plotted using the system parameters that were derived by Horne et al. (1991): an inclination $i=81^{\circ}$, mass ratio $q=0.15$, and $M_{1}=0.61 \mathrm{M}_{\odot}$. We note that the classical two-dimensional visualisation of tomograms used in Fig. 6 does not always provide adequate representation of the multicomponent structure that we discuss below. We find it useful to also present the Doppler map of $\mathrm{H} \alpha$ from the set-2005-n2 in threedimensional (3D) form (Fig. 8).

All the Doppler maps display a ring of disc emission, the radius of which is different for various lines, reflecting the varied peak-to-peak velocity separation in these lines. However, the detailed appearance of the Balmer and helium tomograms is rather dissimilar. Most of the maps show a compact emission area in the fourth quadrant $\left(-V_{x},+V_{y}\right)$, which can be unequivocally identified as the hotspot that is located in the region of interaction between the gas stream and the outer edge of the accretion disc. This area is a dominant emission source in $\mathrm{H} \beta$ and $\mathrm{He} \mathrm{I}$ lines, but very weak in $\mathrm{H} \alpha$. Instead, the $\mathrm{H} \alpha$ map exhibits a bright enhanced emission region in the second quadrant $\left(+V_{x},-V_{y}\right)$ whose ambiguous nature we discuss in Sect. 5.3. The 3D Doppler maps of highest quality $(\mathrm{H} \alpha, \mathrm{H} \beta, \mathrm{He}$ I 25876$)$ clearly show that the hotspot is located on the top of azimuthally extended emission structure of spiral shape (Fig. 8). This spiral structure in the fourth quadrant and the emission region in the second quadrant are disjointed from each other by gaps in the upper- and lowerleft parts of the tomograms. The Fe II $\lambda 5169$ line shows no clear evidence for either of the three emission sources. No sign of the secondary star is seen in either tomogram.

To examine the stability of the emission structure of HT Cas over time, we calculated Doppler maps for the four Balmer and one HeI lines from other available data sets (Fig. 7). Even though the quality of these data is notably worse than of the 2005 data set, they show the same features in Doppler maps. The main dissimilarity between observations is a different contribution of the above-mentioned components.

\subsection{Radial velocity study}

To the best of our knowledge, there was only one attempt to estimate the radial velocity semi-amplitude of the white dwarf in HT Cas in the past. Young et al. (1981) measured the $K_{1}$ velocity of $115 \pm 6 \mathrm{~km} \mathrm{~s}^{-1}$ for the emission lines, but the resulting radial velocity curve is $30^{\circ}$ out of phase with the WD. Horne et al. (1991) showed that this result is inconsistent with most of the photometric data and predicted $K_{1}$ velocity of $58 \pm 11 \mathrm{~km} \mathrm{~s}^{-1}$.

We measured the radial velocities of the emission lines in HT Cas by applying the double-Gaussian method described by Schneider \& Young (1980) and refined by Shafter (1983). This technique consists of convolving each spectrum with a pair of Gaussians of width $\sigma$, the centres of which have a separation of $\Delta$. The position at which the intensities through the two Gaussians become equal is a measure of the wavelength of the emission line. The measured velocities depend on the choice of $\Delta$ and, by varying its value, different parts of the lines can be sampled. It is commonly believed that the most reliable parts of the emission-line profile for deriving the radial velocity curve are the extreme wings. Young et al. (1981) used $\Delta=3200 \mathrm{~km} \mathrm{~s}^{-1}$ in their measurements.

To test for consistency in the derived velocities and the zero phase, we separately used the lines $\mathrm{H} \alpha$ and $\mathrm{H} \beta$ in the set-2005-n1, and $\mathrm{H} \alpha$ in the set-2005-n2. The measurements were made using the Gaussian $\sigma$ of 100 and $200 \mathrm{~km} \mathrm{~s}^{-1}$ and different values of the Gaussian separation $\Delta$ ranging from $1200 \mathrm{~km} \mathrm{~s}^{-1}$ to $4000 \mathrm{~km} \mathrm{~s}^{-1}$ in steps of $50 \mathrm{~km} \mathrm{~s}^{-1}$, following the technique of "diagnostic diagrams" (Shafter et al. 1986). For each value of $\Delta$ 

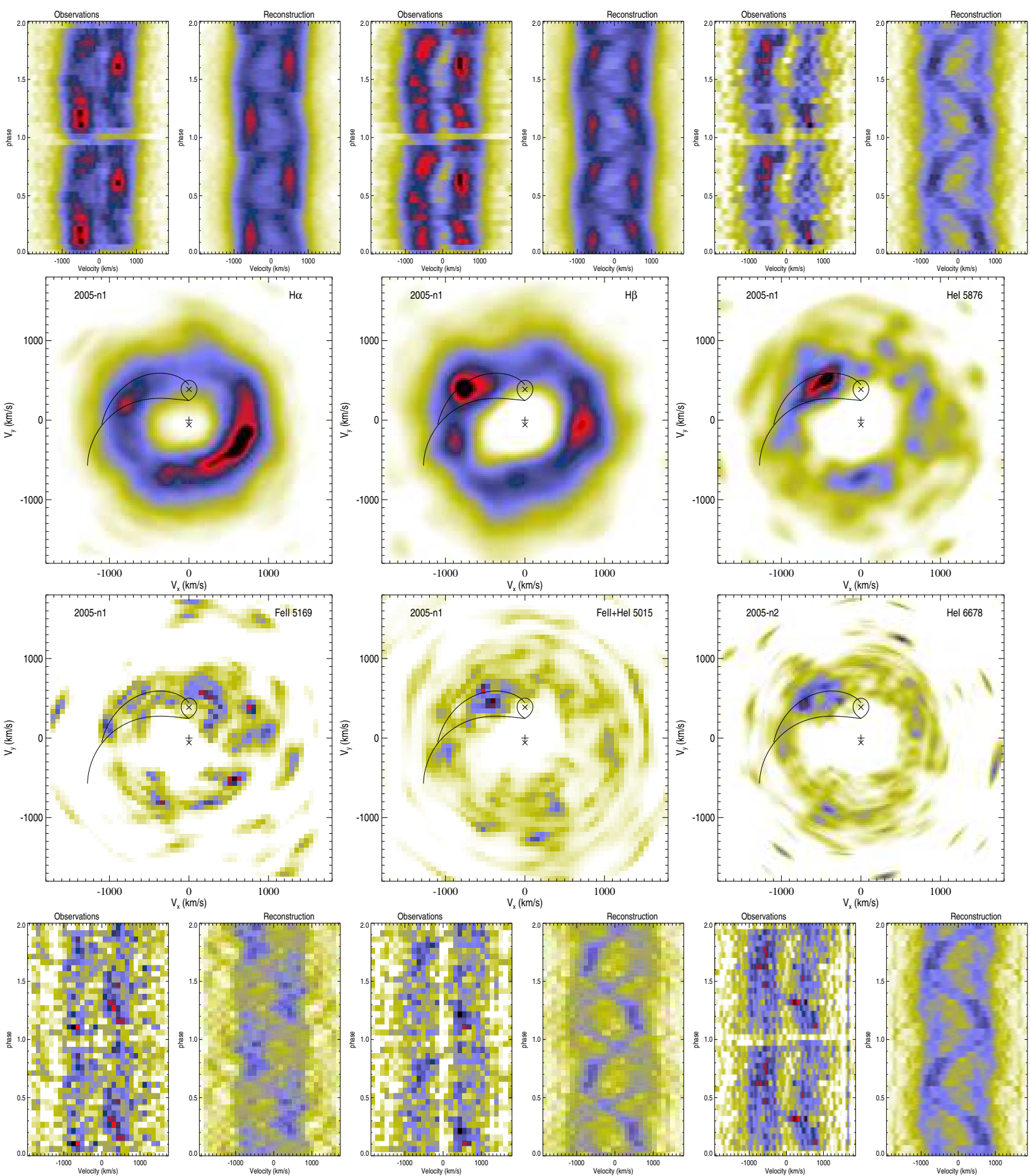

Fig. 6. Doppler tomography for the $\mathrm{H} \alpha, \mathrm{H} \beta$, and He I 5876 emission lines (in the upper half of the figure), and for Fe II 5169, He I 5015, and He I 6678 (in the bottom half of the figure) from the data sets 2005-n1 and 2005-n2. For each line, the Doppler maps (two middle panels) and corresponding observed and reconstructed trailed spectra (top and bottom panels) are shown. Indicated on the maps are the positions of the WD (lower cross), the centre of mass of the binary (middle cross) and the Roche lobe of the secondary star (upper bubble with the cross). The predicted trajectory of the gas stream and the Keplerian velocity of the disc along the gas stream have also been shown in the form of the lower and upper curves, respectively. The Roche lobe of the secondary and the trajectories have been plotted using the system parameters, derived by Horne et al. (1991).

we made a non-linear least-squares fit of the derived velocities to sinusoids of the form:

$V(\varphi, \Delta)=\gamma(\Delta)-K_{1}(\Delta) \sin \left[2 \pi\left(\varphi-\varphi_{0}(\Delta)\right)\right]$

where $\gamma$ is the systemic velocity, $K_{1}$ is the semi-amplitude, $\varphi_{0}$ is the phase of inferior conjunction of the secondary star, and $\varphi$ is the phase calculated according to the ephemeris from
Feline et al. (2005). During this fitting procedure we omitted spectra that covers the phase ranges $\varphi= \pm 0.1$, owing to measurement uncertainties during the eclipse.

The resulting diagnostic diagrams are shown in Fig. 9. The diagrams show the variations of $K_{1}, \sigma\left(K_{1}\right) / K_{1}$ (the fractional error in $\left.K_{1}\right), \gamma$, and $\varphi_{0}$ with $\Delta$ (Shafter et al. 1986). To derive the orbital elements of the line wings, Shafter \& Szkody (1984) 
V. V. Neustroev et al.: Multi-epoch tomographic study of HT Cas
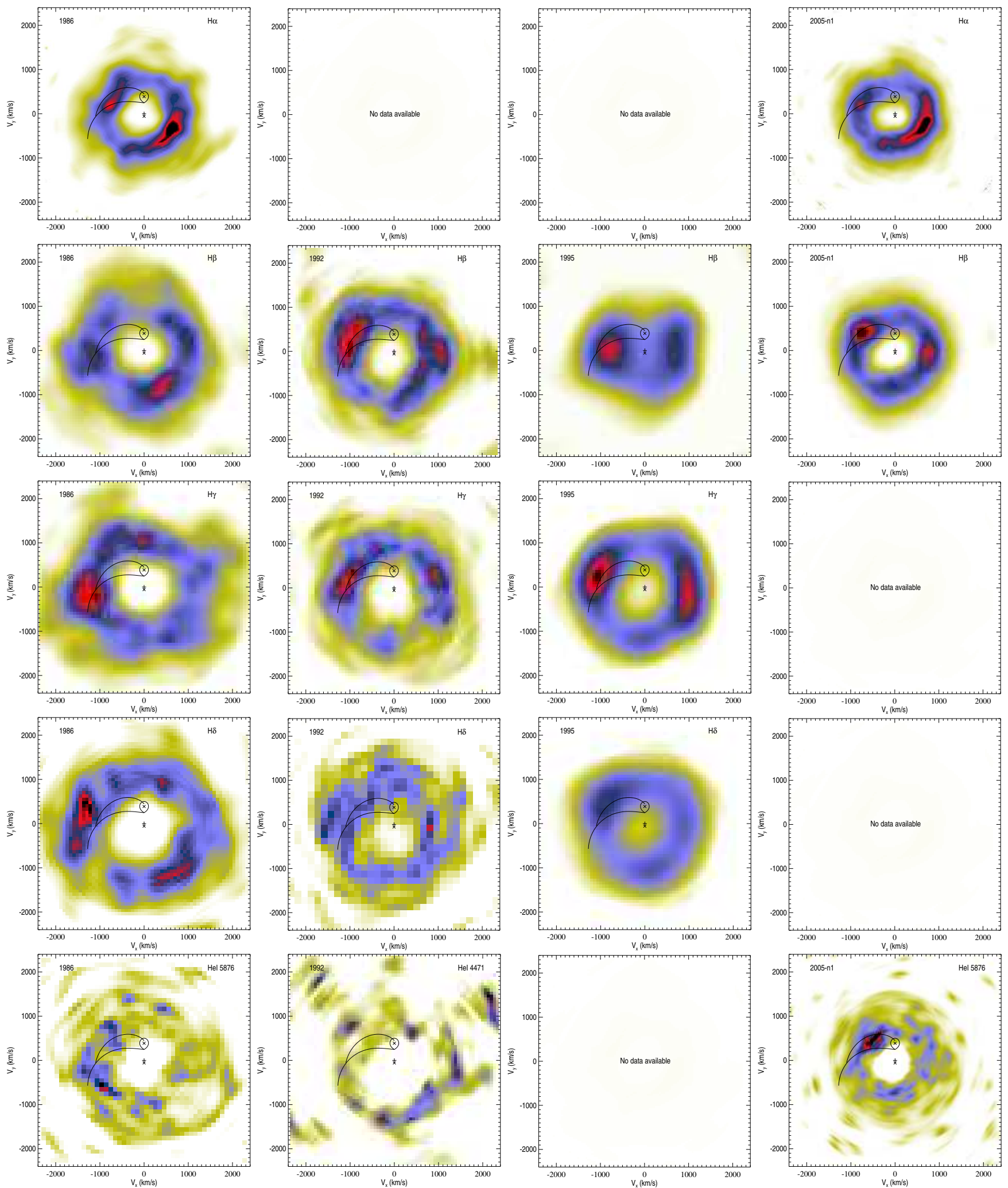

Fig. 7. Doppler tomography for the Balmer and He I emission lines. Each column shows the maps for different data sets (1986, 1992, 1995 and 2005-n1). Each row shows the maps for different lines ( $\mathrm{H} \alpha, \mathrm{H} \beta, \mathrm{H} \gamma, \mathrm{H} \delta$ and $\mathrm{He} \mathrm{I})$.

suggest taking the values that correspond to the largest separation $\Delta_{\max }$, just before $\sigma\left(K_{1}\right) / K_{1}$ shows a sharp increase. We note, however, that all the parameters are consistent for different lines and are quite stable over a reasonable range of Gaussian separations around $\Delta_{\max }$ which can be set at $\sim 2500-2700 \mathrm{~km} \mathrm{~s}^{-1}$. Using $\sigma\left(K_{1}\right), \sigma(\gamma)$ and $\sigma\left(\varphi_{0}\right)$ as a weight factor, we find the following mean values of the orbital patameters: $K_{1}=61 \pm 8 \mathrm{~km} \mathrm{~s}^{-1}, \gamma=-9 \pm 5 \mathrm{~km} \mathrm{~s}^{-1}$, and $\varphi_{0}=0.15 \pm 0.02$.

The derived value of $K_{1}$ is very much consistent with the one predicted by Horne et al. (1991). However, the radial velocity curves of all the investigated emission lines are significantly 

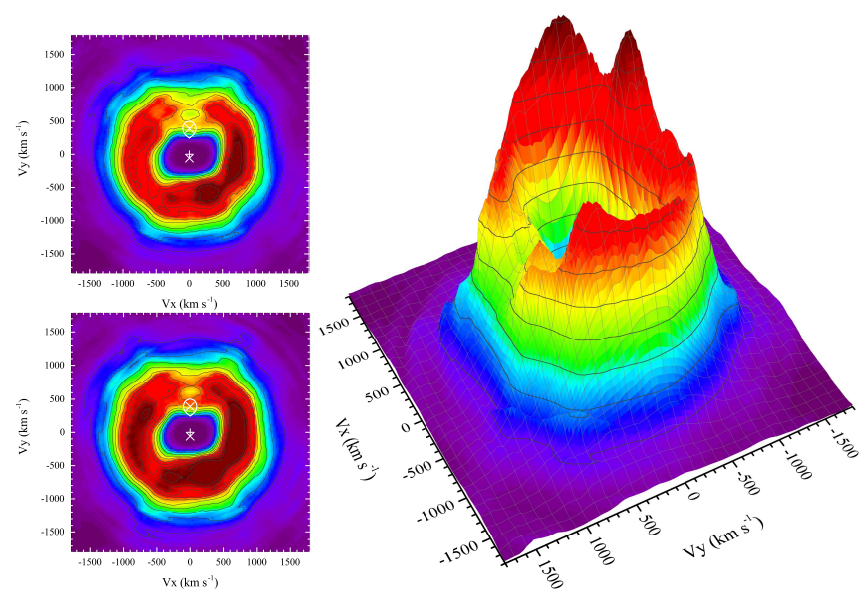

Fig. 8. Doppler map of the $\mathrm{H} \alpha$ emission line from the set-2005-n2 in $3 \mathrm{D}($ right $)$ and 2D representations with different contrasts to emphasise various components of the map (left).

shifted relative to the eclipse $\left(\sim 55^{\circ}\right)$, and therefore these lines cannot be used to represent the motion of the WD. As seen in the diagnostic diagrams (Fig. 9), the shift is observed over the whole range of separations $\Delta$, from emission-line profile peaks to the extreme wings where the noise begins to dominate. This shift is almost twice as large as that reported by Young et al. (1981). Although the Doppler maps of HT Cas have a very complex structure, they show no evidence for compact emission or absorption sources at the far wings of spectral lines, which can be responsible for the observed phase shift in this velocity range $\left(>1500 \mathrm{~km} \mathrm{~s}^{-1}\right)$. This fact suggests that a global asymmetric configuration may exist in the inner parts of the accretion disc of HT Cas (e.g. eccentric, elliptical structure).

\section{Discussion}

A Doppler tomography technique applied to a large set of multiepoch spectroscopic observations of HT Cas reveals an unusual distribution of emission in this system. The tomograms show at least three areas of enhanced emission: the hotspot superposed on the spiral structure in the fourth quadrant, and the broad, extended bright region of uncertain origin in the second quadrant.

To explain this structure, we need to assume its spatial location in the binary system, but this is not possible without a detailed knowledge of the velocity field. Although the standard Shakura-Sunyaev model assumes that the gas in the accretion disc moves with circular Keplerian velocities (Shakura \& Sunyaev 1973), this assumption is somewhat aprioristic and it is still unclear if the velocity field of accretion discs is actually Keplerian. Furthermore, this assumption may not be accurate in a strict sense, because some of the system components, such as the gas stream do not follow a pure Keplerian rotation law (Marsh et al. 1990). The formation of spiral shocks in the hot discs of outbursting CVs may also cause deviations from Keplerian motion. Indeed, Baptista et al. (2005) presented evidence that the velocity of the emitting gas along the spiral pattern in the accretion disc of IP Peg in outburst is lower than the Keplerian velocity for the same disc radius.

On the other hand, we find that the Keplerian assumption gives an acceptable working basis, since no significant deviation from the Keplerian rotation has been ever reported for quiescent accretion discs. Instead, evidence of material in Keplerian rotation has been obtained by many authors. For example, Marsh (1988) found strong evidence for Keplerian rotation of

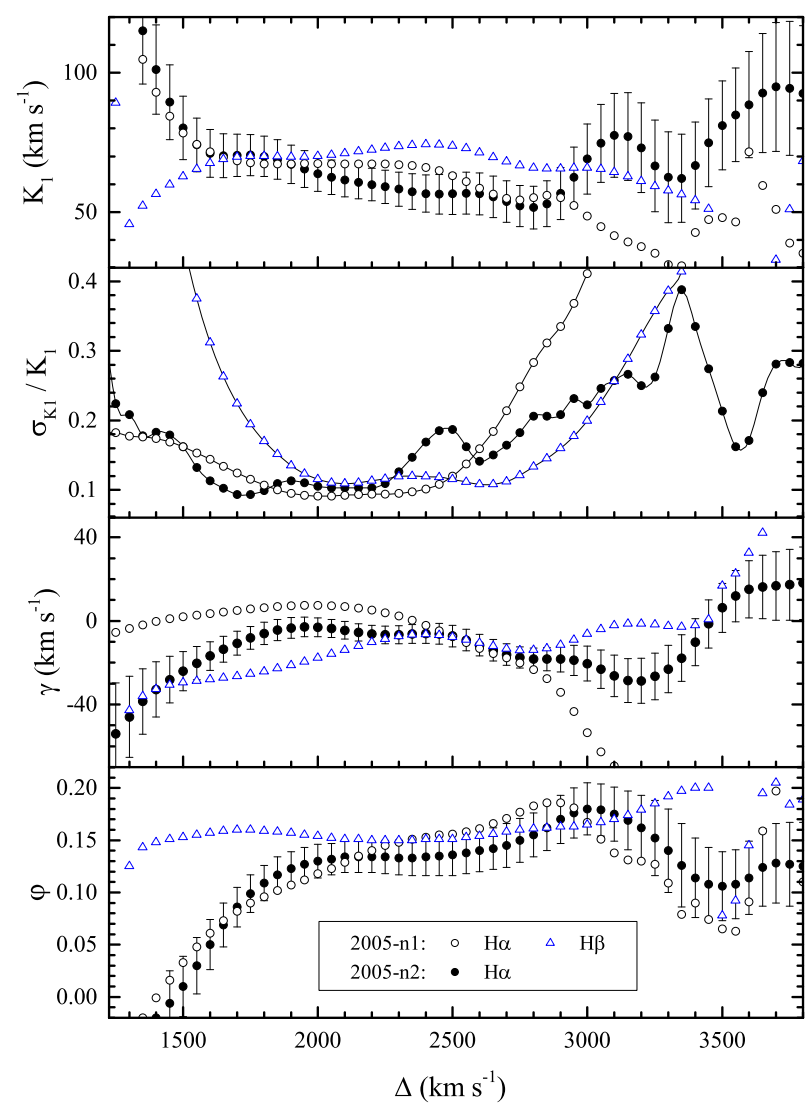

Fig. 9. Diagnostic diagram for the $\mathrm{H} \alpha$ and $\mathrm{H} \beta$ emission lines from the sets 2005-n1 and 2005-n2, showing the response of the fitted orbital elements to the choice of the double-Gaussian separation.

the quiescent disc of above-mentioned IP Peg, the only detected deviations being caused by the stream. Ishioka et al. (2004) also conclude that the behaviour of the disc of IP Peg is basically consistent with Keplerian rotation, though more complex than those predicted by a simple axisymmetric model. In the particular case of HT Cas, Young et al. (1981) presented a crude verification that the disc in this system is Keplerian over at least a factor of 4 in radius. Bearing these considerations in mind, we thus find it useful to create and show, for illustration purposes and to facilitate further discussion, a map of the $\mathrm{H} \alpha$ emission in spatial coordinates, assuming a circular Keplerian flow in the disc (Fig. 10).

The accretion disc, as it appeared in Fig. 10, looks rather unusual. A general impression is that the disc of HT Cas is indeed patchy, as was suggested by Vrielmann et al. (2002). We discuss the derived structure in the following subsections.

\subsection{Hotspot and the size of the accretion disc}

Assuming the Keplerian velocity in the accretion disc, the measured projected outer disc velocity $V_{\text {out }}$ can be used to determine the radius of the disc:

$R_{\mathrm{d}}=\frac{G M_{1} \sin ^{2} i}{V_{\text {out }}^{2}}$.

Individual spectral lines are sensitive to the local physical conditions in the disc (e.g. gas density and temperature), so different lines may lead to different apparent disc radii, which reflects the region where that particular line is excited. We measured $V_{\text {out }}$ 


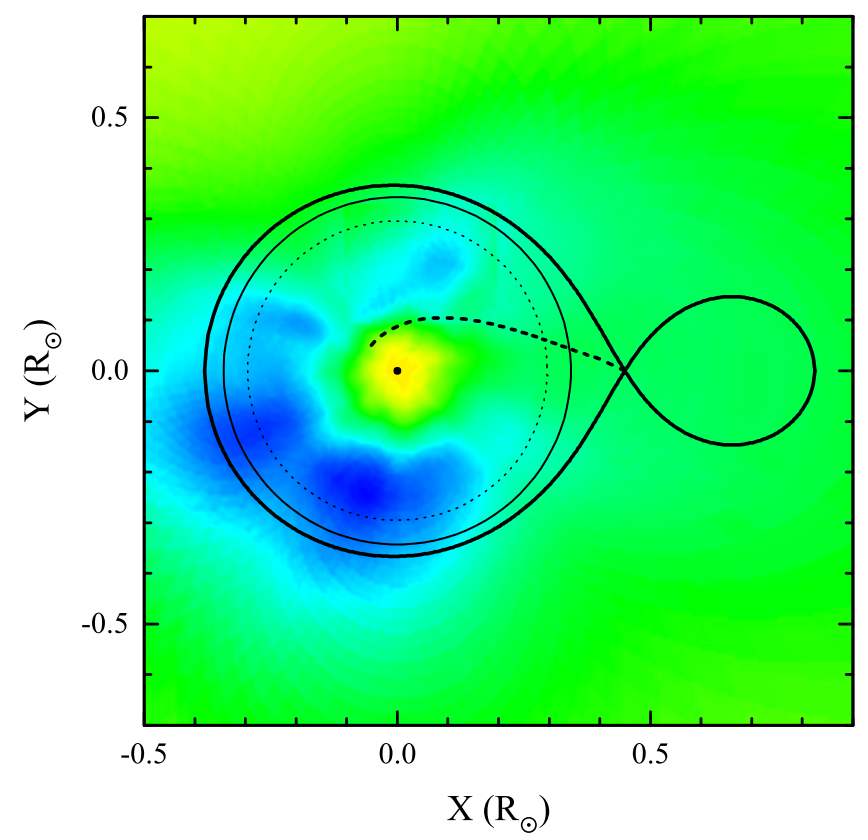

Fig. 10. $\mathrm{H} \alpha$ Doppler map from the 2005 observations transformed to a spatial image, assuming a circular Keplerian flow in the disc. The Roche lobes of the stars (thick solid line), the tidal truncation (thin solid line), and 3:1 resonance (thin dashed line) radii are also shown. The thick dashed line represents the gas stream from the donor star.

through the modelling of the $\mathrm{H} \alpha$ emission line that has a relatively low excitation energy and that has been proven to be a good tracer of the cool outer parts of the accretion disc. If $\mathrm{H} \alpha$ is not present in the given data set, we used the $\mathrm{H} \beta$ line (see Sect. 4.3 and Table 2). Firstly, we used the 2005 data, for which we adopted the value for $V_{\text {out }}$ as being $575 \pm 4 \mathrm{~km} \mathrm{~s}^{-1}$. This value is the weighted mean for the $\mathrm{H} \alpha$ and $\mathrm{H} \beta$ lines from the two nights, using $\sigma\left(V_{\text {out }}\right)$ as a weight factor. Using the system parameters derived by Horne et al. (1991), this value gives $R_{\mathrm{d}} \approx 0.52 \pm 0.01 a$.

The obtained disc radius coincides precisely with the tidal truncation radius of the accretion disc $r_{\max }=0.522 a$ (for $q=$ 0.15), which can be estimated using Equation 2.61 from Warner (1995)

$r_{\max }=a \frac{0.6}{1+q}$.

Such a large disc is slightly tidally distorted and elongated perpendicular to the line of centres of the WD and the secondary star. This means that some deviations from circular Keplerian flow are expected at the outer disc. The magnitude of these deviations were estimated by different methods with roughly consistent results. For example, Steeghs \& Stehle (1999) showed, using 2D hydrodynamic calculations, that the departures can reach $100 \mathrm{~km} \mathrm{~s}^{-1}$, which is in agreement with the inviscid (noninteracting single particle) calculations of Paczynski (1977). Because of this effect, the peak-to-peak separation of emissionline profiles is also expected to vary as a function of binary phase, which affects the disc radius measurement. This variation can, in principle, be measured using time-resolved spectroscopy. Unfortunately, we failed to either confirm or deny this effect with our medium-resolution data. Individual line profiles in our spectra are relatively noisy and they are significantly affected by small- and large-scale structures in the accretion disc, which are difficult to take into account. However Steeghs \& Stehle (1999) pointed out that, for the orbit averaged spectra, the velocity deviations mostly cancel out, so the assumption of circular Keplerian velocities is still reliable. Nevertheless, to be on the conservative side, we assume that the average disc size measured from the orbit averaged profiles is accurate to better than $10 \%$. Thus, for HT Cas we adopt $R_{\mathrm{d}}=0.52 \pm 0.05 a$.

A comparison of the peak-to-peak separation of emission lines from different observations, including those presented by Young et al. (1981), shows that such a large disc seems to be normal for HT Cas. Most of the data (except for the $1992 \mathrm{ob}-$ servations) suggest that the accretion disc radius $R_{\mathrm{d}}$ lies within the range $0.45-0.52 a$. This result is not consistent with previous radius measurements. As we mentioned in Sect. 2, a typical value of $R_{\mathrm{d}}$ in quiescence was measured as just $\sim 0.23 \mathrm{a}$. We note that most of the measurements found in the literature were based on the position of the hotspot. Among the methods used were the eclipse-mapping technique (Feline et al. 2005) and methods based on contact timings of the bright spot eclipses (Horne et al. 1991), and on modelling the eclipse data (Zhang et al. 1986). Our data, however, indicate that the hotspot is not located at the accretion disc edge.

Figure 11 (left-hand panel) shows a zoomed hotspot area of the map, which combines all the tomograms from the 1986 and 2005 observations. The position of the hot spot in all the emission lines from 2005 is consistent with the trajectory of the gas stream and either has a velocity close to the expected velocity of the stream at a distance from the WD (i.e., $\mathrm{H} \alpha$ ), or a Kepler velocity at the stream position $(\mathrm{H} \beta, \mathrm{He}$ I 6678$)$, or a mix of these velocities (He I 5876). In 1986 the $\mathrm{H} \alpha$ spot is seen in nearly the same position as in 2005, whereas all other lines show the spot (marked by a grey oval) somewhat further along the stream trajectory. The latter spot is also evident in Doppler maps from other data sets. It is interesting that all the peaks of spot emission are located much closer to the WD $\left(R_{\mathrm{hs}} \approx 0.22-0.30 a\right)$ than the disc edge that was measured from the double-peaked profiles $\left(R_{\mathrm{d}} \approx 0.52 a\right)$. This suggests that the gas stream flows almost unaffectedly through the outer disc regions before it starts to be seen as a continuum and line-emission source. A similar finding is reported by Skidmore et al. (2000) and Mason et al. (2000) for the dwarf nova WZ Sge.

Such a low density outer disc region is expected to have much lower optical depth than the inner disc, which is in agreement with the rather steep Balmer decrement to be observed in HT Cas. We also note the work of Vrielmann et al. (2002) who perform a Physical Parameter Eclipse Mapping analysis of multicolour photometric observations of HT Cas and show that the accretion disc is moderately optically thin, but becomes nearly optically thick near the WD. They found that in the $R$ band, the disc region outside $\sim 0.28 a$, which they call the disc edge, contributes less than $1 \%$ of the flux compared to the WD. We point out that, in fact, this edge is the photometric one, after which the accretion disc gas does not produce much broadband continuum light. The spectroscopic data suggest, however, that optically thin gas, visible in spectral lines, is extended further than the photometric disc. There is also evidence that such disagreement between photometric and spectroscopic sizes of the accretion disc can be observed not only in quiescence, but also in outbursts (Isogai et al. 2015).

\subsection{Spiral arm in the trailing side of the accretion disc}

The weakness of the hot spot in the $\mathrm{H} \alpha$ line makes it possible to trace the extended emission structure in the fourth quadrant of the tomograms in detail. Figure 11 (right-hand panel) shows 

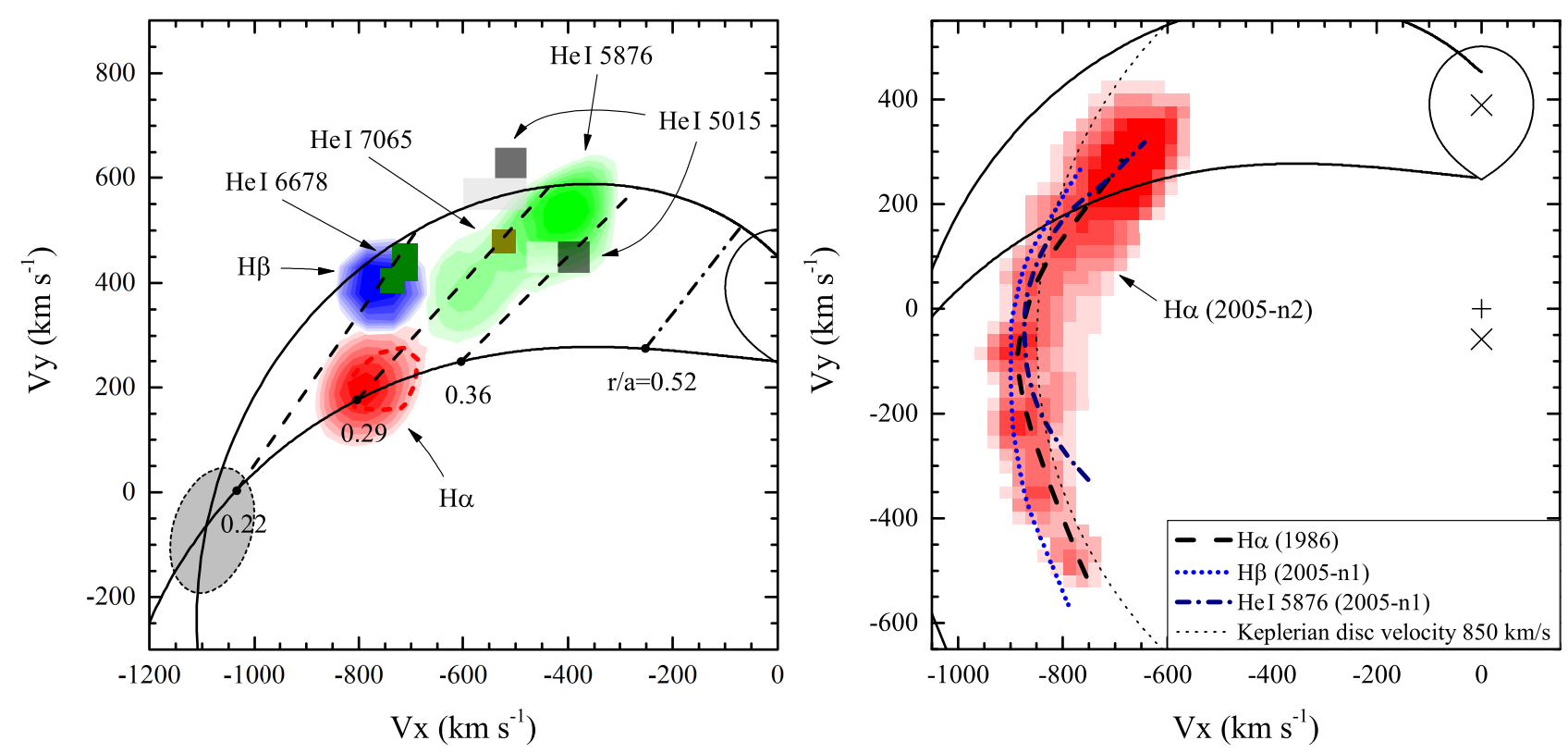

Fig. 11. Left: zoomed hotspot area of the Doppler map combined from all the tomograms taken during the 2005 observations, with different contrasts to emphasise the hotspot location. The map also shows the spot location from the 1986 observations. The H $\alpha$ spot is marked by the red dashed line. A grey oval shows the other spectral lines. The dashed lines connect the velocity of the ballistic gas stream (lower curve) and the velocity on the Keplerian disc along the gas stream (upper curve) for the same points at distances labelled along the lower curve (in $r / a$ units). The dash-dotted line corresponds to the measured radius of the accretion disc, which coincides with the tidal truncation radius $r_{\max } / a=0.52$. Right: zoomed part of the $\mathrm{H} \alpha$ Doppler map from the set-2005- $\mathrm{n} 2$ centred around the spiral arm area. The thick lines show the trace of this area in other spectral lines and data sets. Dotted thin line shows the Keplerian disc velocity of $850 \mathrm{~km} \mathrm{~s}^{-1}$.

a zoomed part of the $\mathrm{H} \alpha$ Doppler map from the set-2005-n2 that is centred around this area. The latter can also be traced in other spectral lines and data sets, some of which are denoted in Fig. 11 (right-hand panel) by different lines. The location and shape of the structure is nearly the same in all the lines. It starts at the hotspot area and extends downstream in azimuth for $\sim 60^{\circ}$. Its width gradually decreases until this "tail" has completely disappeared.

The origin of this emission is not clear. A similar structure was observed, e.g. in WZ Sge (Spruit \& Rutten 1998) and U Gem (Unda-Sanzana et al. 2006). This kind of tail cannot be due to stream-disc overflow, which might produce an excess of emission along the path of the stream (Kunze et al. 2001). On the other hand, a tail is to be expected as a consequence of the postimpact hydrodynamics of the gas stream and could be caused by material that has settled into Keplerian motion downstream from the hotspot (for a discussion see Spruit \& Rutten 1998, and references therein). However, despite an overall similarity in the tails in HT Cas and WZ Sge, they are significantly different. The tail in WZ Sge indeed shows circular Keplerian velocities along its trail, whereas the velocity in HT Cas increases from $\sim 760$ to $880 \mathrm{~km} \mathrm{~s}^{-1}$ (Fig. 11, right-hand panel). This corresponds to the range of distances from the WD from $0.30 a$ to $0.22 a$ (Fig. 10). In fact, this type of behaviour resembles the signature generated by spiral waves in the disc, rather than the hotspot tail in WZ Sge. However, the properties of spiral structure in the accretion discs have been particularly well studied by both numerical simulations and observations (Steeghs \& Stehle 1999, and references therein), and no spiral waves or shocks are predicted in quiescent discs.

The observed spiral feature could be associated with a tidally thickened sector of the disc that is elevated owing to tidal distortions and being irradiated by the WD or inner disc (for a discussion see Ogilvie 2002; Unda-Sanzana et al. 2006, and Sect. 5.3 below), although this type of thickening is more likely in the outer disc than at the observed position. Another possible explanation for the spiral arm in HT Cas may come from the hydrodynamical simulations by Bisikalo et al. (1998). They showed that the gas stream may penetrate the outer disc regions, producing a so-called hot line (an extended shock wave) that interacts and mixes with the disc, allowing matter to be deposited at the inner disc regions.

\subsection{Emission region in the leading side of the accretion disc}

From Figs. 6 and 7, it may appear that the emission region in the second quadrant of tomograms is bright in the $\mathrm{H} \alpha$ line only, much weaker in $\mathrm{H} \beta$, and almost undetectable in the He I lines. This impression is somewhat misleading because of the chosen contrast and colour scaling to emphasise different components of Doppler maps. We estimated the contribution of this emission source to the total flux of strongest emission lines from the set$2005(\mathrm{H} \alpha, \mathrm{H} \beta$, and $\mathrm{He}$ I 5876) and set-1986 $(\mathrm{H} \alpha$ and $\mathrm{H} \beta)$ and found nearly the same value of 5-7 per cent for all of them. The region trails along the accretion disc ring for some $150^{\circ}$ in azimuth and perhaps has a multicomponent structure. The highest quality and resolution Doppler map of $\mathrm{H} \alpha$ from the set-2005-n2 (Fig. 8) clearly shows two distinct spots of similar brightness - in the bottom and lower-right sides of the disc ring - while in some of the other tomograms one of these two spots prevails over the other.

We compared the location of these structures to those found in different lines from the 2005 observations and from different sets of observations. Figure 12 shows that at least the lower-right spot is always observed in the same position (there is a weak sign of vertical shift for the bottom spot), even though the accretion disc properties differ considerably from one set of observations to another (see Sect. 4.1). This resembles the behaviour of another short-period CV, namely BZ UMa, in which the similar spot in the leading side of the accretion disc remained present at 
V. V. Neustroev et al.: Multi-epoch tomographic study of HT Cas
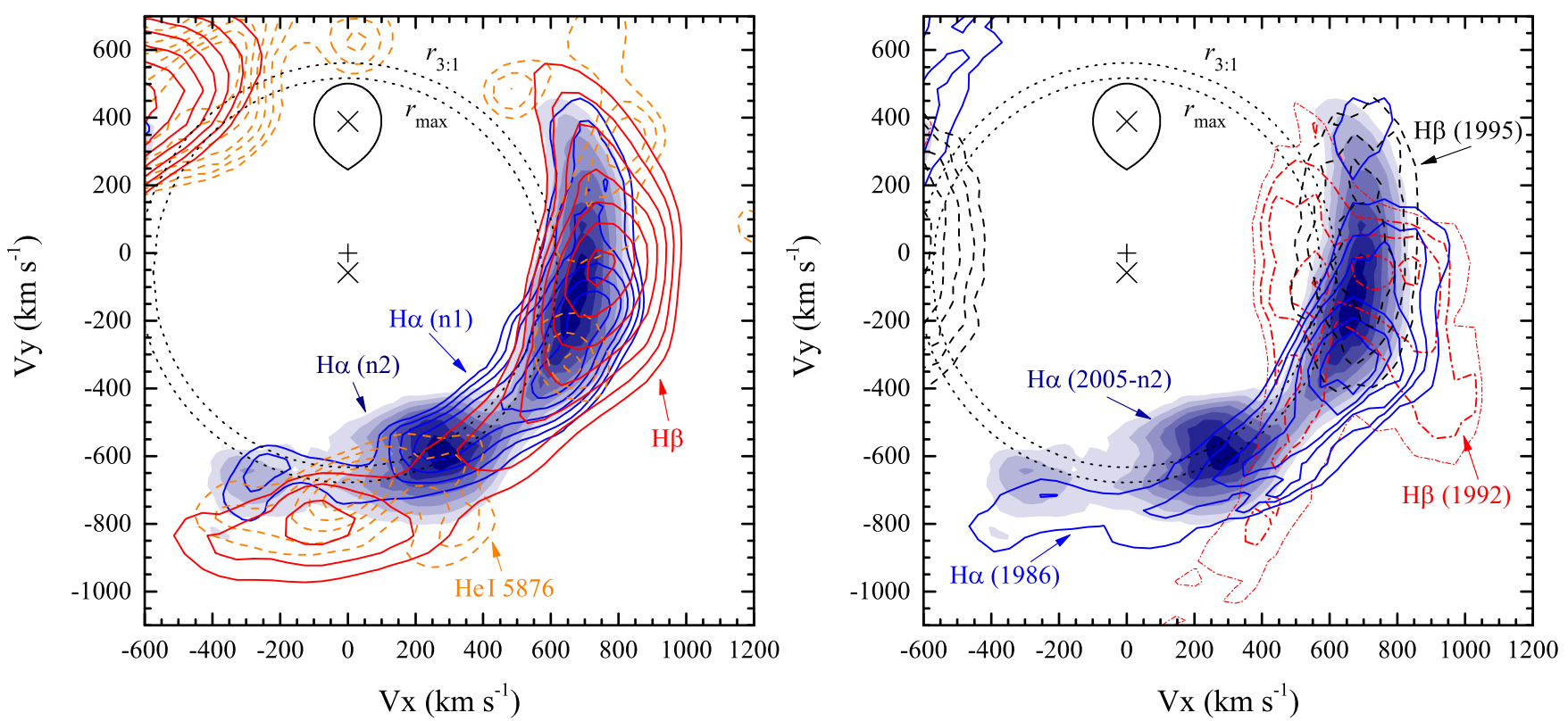

Fig. 12. Doppler maps that combine the tomograms for different lines from the 2005 observations (left) and for the strongest lines from different sets of observations (right). The maps are zoomed around the emission region in the leading side of the accretion disc. The circular dashed lines represent Keplerian velocities at the tidal truncation $\left(r_{\max }\right)$ and 3:1 resonance $\left(r_{3: 1}\right)$ radii.

the same position during all stages of the outburst, from quiescence to the maximum (Neustroev et al. 2006). The velocities of the emission region suggest its origin in the outer accretion disc. The spatial map of HT Cas indicates a relatively sharp inner border of the region at $R_{\mathrm{bs} \text {,in }} \approx 0.25 a$, whereas its outer parts can be traced up to the Roche lobe radius exceeding $r_{\max }$ (Fig. 10). Though the latter property may well be an artefact of reconstruction because of the finite spectral resolution, there is no doubt that the emission comes from the outermost parts of the disc.

Being located at the opposite side of the accretion disc, neither of the bright spots can be associated with the interaction between the gas stream and the disc. No shock waves that can produce an excess of emission in the bottom-right side of tomograms are predicted by hydrodynamical simulations, and none of the theories foresee stable shocks in the quiescent accretion disc. We propose that the leading side bright spots are caused by irradiation of relatively compact thickened sectors of the outer disc by the WD and/or hot, inner disc regions. The reason for this thickening is not clear, but can be assumed to be tidally induced. A clue to understanding the exact process can perhaps be gained from the fact that the outer parts of a large accretion disc are under the gravitational influence of the secondary star. This prevents the disc from growing above the tidal radius $r_{\max }$, where the tidal and viscous stresses are comparable (Warner 1995). How that truncation occurs and how the disk thickness varies along the outer edge has not yet been well established. To the best of our knowledge, no detailed 3D numerical simulations have been devoted to these questions so far. However, Bisikalo et al. (1998) pointed out an important role of the circum-disc halo that is created by matter which went outside $r_{\max }$ and left the accretion disc. In their hydrodynamical simulations, the accretion disc has a quasi-elliptical shape that extended in a direction opposite to the hot spot (see also Kononov et al. 2012). Truss (2007) also reported the appearance of similarly oriented elliptical discs, although only in extreme mass-ratio compact binaries $(q<0.1)$.

The asymmetry of the disc can also explain the shift of the radial velocity curve relative to the inferior conjunction of the secondary star, as detected in HT Cas (Sect. 4.5) and many other short-period CVs (Mason et al. 2000). The fact that the shift is observed in a wide range of distances from the WD suggests significantly asymmetric structures exist, even in the inner parts of the disc.

\subsection{Large accretion discs in cataclysmic variables}

The discovery of the extended bright area in the leading side of the accretion disc of HT Cas enriches the list of objects in which this type of feature has been observed. Based on the large measured radius of the disc in HT Cas, we make a guess in the previous subsection that the leading side bright spot was caused by tidally induced thickened sectors of the outer large disc. In this respect it might be worth examining if other systems that show a similar emission feature also have the large accretion disc.

We inspected several CVs with relatively well measured system parameters from the list given in the Introduction, and found that most of them support our idea. For example, the disc in VW Hyi appears slightly larger than the tidal radius $r_{\max }$ (Smith et al. 2006), whereas in WZ Sge, V406 Vir, EZ Lyn, and the old nova RR Pic, the disc radius was shown to be no smaller than the 3:1 resonance radius (Skidmore et al. 2000; Aviles et al. 2010; Zharikov et al. 2013; Schmidtobreick et al. 2008). The disc radius of IP Peg, as estimated from the double-peaked emission line profiles ( $\sim 570 \mathrm{~km} \mathrm{~s}^{-1}-$ Neustroev et al. 2002), also appears to be a bit larger than the tidal truncation radius $(0.45 \pm 0.05 a$ and $0.40 a$, respectively).

Thus, most of the CVs with the leading side bright spot have the accretion disc, the radius of which is close to the tidal truncation limit. It is worth noting that, at least in a few of them, the radius changes little with time. For example, Mason et al. (2000) claim that the same accretion disc radius has been observed in WZ Sge for 40 years. Our data also demonstrate that the radius of the disc in HT Cas has not changed much during all our observations, remaining consistently large. This contradicts the modern understanding of the evolution of the accretion disc through an outburst cycle, according to which the disc expands 
during the outburst and then contracts with time (Warner 1995). The conclusion, that the disc radius in many short-period CVs is close or even larger than the 3:1 resonance radius, has important implications that relate to the observational properties of such systems. It is expected that, when the accretion disc expands beyond the 3:1 resonance radius, this causes the disc to become quasi-elliptical and precess. The enhanced tidally-driven viscous dissipation in the disc, varying on the beat between the orbital and disc precession periods, can result in superhumps in the light-curve (Osaki 1989). Thus, if it occurs during the quiescent state, then quiescent superhumps can be present. However, the detectability of such superhumps should very much depend on the physical conditions in the outer disc. For instance, it is difficult to expect strong quiescent superhumps in the lightcurve of HT Cas, whose outer accretion disc contributes a negligible amount of the total broadband light.

\section{Summary}

We have presented multi-epoch, time-resolved optical spectroscopic observations of the dwarf nova HT Cas, obtained during 1986, 1992, 1995, and 2005, with the aim of studying the properties of emission structures in the system. Though HT Cas has always remained in quiescence, its mean brightness has changed substantially between the observations. The spectra are dominated by very strong and broad double-peaked emission lines of the Balmer series. Numerous weaker lines of neutral helium and singly ionized iron (Fe II) are also present. The high-excitation line of He II $\lambda 4686$ is clearly detected. A comparison of the averaged spectra from different data sets shows significant quantitative differences between them. There are notable variations in both emission-line strengths and their ratios for different lines, which indicates a variable mix of optically thin and thick conditions. Nevertheless, these variations do not correlate with the system flux.

The emission lines are very broad with a FWZI of up to 5-6 thousand $\mathrm{km} \mathrm{s}^{-1}$ and a peak-to-peak separation of $\gtrsim 1100 \mathrm{~km} \mathrm{~s}^{-1}$. We determined that the accretion disc radius, measured from the double-peaked profiles, is consistently large and lies within the range of $0.45-0.52 a$. This is close to the tidal truncation radius $r_{\max }=0.52 a$ and slightly larger than the 3:1 resonance radius $r_{3: 1}$ of $0.45 a$. This result is not consistent with previous radius measurements.

The radial velocity semi-amplitude of the WD was found to be $K_{1}=61 \pm 8 \mathrm{~km} \mathrm{~s}^{-1}$ from the motion of the wings of the emission lines. This value is very consistent with that predicted by Horne et al. (1991). However, the radial velocity curves of all the investigated emission lines are significantly shifted relative to the eclipse $\left(\sim 55^{\circ}\right)$, and therefore these lines cannot be used to represent the motion of the WD. The shift is observed from the profile peaks to the extreme wings, suggesting a global asymmetry may exist in the accretion disc of HT Cas.

An extensive set of Doppler maps has revealed a very complex emission structure of the accretion disc. Apart from a ring of disc emission, the tomograms display at least three areas of enhanced emission: the hotspot from the area of interaction between the gas stream and the accretion disc, which is superposed onto the elongated spiral structure, and the extended bright region on the leading side of the disc, opposite the location of the hotspot.

The position of the hotspot in all the emission lines is consistent with the trajectory of the gas stream. However, the peaks of emission are located in the range of distances $R_{\mathrm{hs}} \approx 0.22-0.30 a$, which are much closer to the WD than the disc edge $(0.52 a)$.
This suggests that the outer disc regions have a very low density, allowing the gas stream to flow almost freely before it starts to be seen as an emission source. The spiral arm appears as a consequence of such a penetration. The stream produces an extended shock wave that interacts and mixes with the disc.

The extended emission region in the leading side of the disc has been observed in many CVs, but it has had no plausible explanation until now. We found that in all the emission lines of HT Cas, this structure is always observed in the same position - at the very edge of the large disc. Observations of other CVs, which show a similar emission structure in their Doppler maps, seem to confirm this conclusion. We propose that the leading side bright region is caused by irradiation of tidally thickened sectors of the outer disc by the WD and/or hot inner disc regions.

Acknowledgements. The authors would like to thank Valery Suleimanov for useful comments and Natalia Neustroeva for help in preparing the manuscript. We are thankful to the anonymous referee for their careful reading of the manuscript. This work was supported by PAPIIT grants IN-100614 and CONACyT grants 151858 , and CAR 208512 for resources provided for this research.

\section{References}

Aviles, A., Zharikov, S., Tovmassian, G., et al. 2010, ApJ, 711, 389

Baptista, R., Morales-Rueda, L., Harlaftis, E. T., Marsh, T. R., \& Steeghs, D. 2005, A\&A, 444, 201

Bạkowska, K., \& Olech, A. 2014, Acta Astron., 64, 247

Bisikalo, D. V., Boyarchuk, A. A., Chechetkin, V. M., Kuznetsov, O. A., \& Molteni, D. 1998, MNRAS, 300, 39

Borges, B. W., Baptista, R., Papadimitriou, C., \& Giannakis, O. 2008, A\&A, 480, 481

Borisov, N. V., \& Neustroev, V. V. 1997, Bull. Special Astrophys. Obs., 44, 110

Drabek, S. V., Kopylov, I. M., Somov, N. N., \& Somova, T. A. 1986, Astrofizicheskie Issledovaniia Izvestiya Spetsial'noj Astrofizicheskoj Observatorii, 22, 64

Feline, W. J., Dhillon, V. S., Marsh, T. R., Watson, C. A., \& Littlefair, S. P. 2005, MNRAS, 364, 1158

Greenstein, J. L., \& Kraft, R. P. 1959, ApJ, 130, 99

Heerlein, C., Horne, K., \& Schwope, A. D. 1999, MNRAS, 304, 145

Hoffmeister, C. 1943, Astron. Nachr., 274, 36

Honeycutt, R. K., Schlegel, E. M., \& Kaitchuck, R. H. 1987, ApJS, 65, 451

Horne, K., \& Marsh, T. R. 1986, MNRAS, 218, 761

Horne, K., \& Saar, S. H. 1991, ApJ, 374, L55

Horne, K., Wood, J. H., \& Stiening, R. F. 1991, ApJ, 378, 271

Ioannou, Z., Naylor, T., Welsh, W. F., et al. 1999, MNRAS, 310, 398

Ishioka, R., Mineshige, S., Kato, T., Nogami, D., \& Uemura, M. 2004, PASJ, 56, 481

Isogai, M., Arai, A., Yonehara, A., et al. 2015, PASJ, 67, 7

Kato, T., Maehara, H., Miller, I., et al. 2012, PASJ, 64, 21

Kniazev, A. Y. 1994, The Control and Data Acquisition System of the Spectrophotometer at the BTA Nasmyth-1 Focus: IV. Reduction of Scanner Data in MIDAS, SAO Report

Kononov, D. A., Giovannelli, F., Bruni, I., \& Bisikalo, D. V. 2012, A\&A, 538, A94

Kraft, R. P., Mathews, J., \& Greenstein, J. L. 1962, ApJ, 136, 312

Kunze, S., Speith, R., \& Hessman, F. V. 2001, MNRAS, 322, 499

Longa-Peña, P., Steeghs, D., \& Marsh, T. 2015, MNRAS, 447, 149

Marsh, T. R. 1988, MNRAS, 231, 1117

Marsh, T. R. 1990, ApJ, 357, 621

Marsh, T. R. 2001, in Astrotomography, Indirect Imaging Methods in Observational Astronomy, eds. H. M. J. Boffin, D. Steeghs, \& J. Cuypers (Berlin: Springer Verlag), Lect. Notes Phys., 573, 1

Marsh, T. R., \& Horne, K. 1988, MNRAS, 235, 269

Marsh, T. R., \& Horne, K. 1990, ApJ, 349, 593

Marsh, T. R., Horne, K., Schlegel, E. M., Honeycutt, R. K., \& Kaitchuck, R. H. 1990, ApJ, 364, 637

Mason, E., Skidmore, W., Howell, S. B., et al. 2000, MNRAS, 318, 440

Neustroev, V. V. 1998, Astron. Rep., 42, 748

Neustroev, V. V., Borisov, N. V., Barwig, H., et al. 2002, A\&A, 393, 239

Neustroev, V. V., Zharikov, S., \& Michel, R. 2006, MNRAS, 369, 369

Neustroev, V. V., Suleimanov, V. F., Borisov, N. V., Belyakov, K. V., \& Shearer, A. 2011, MNRAS, 410, 963

Neustroev, V. V., Veledina, A., Poutanen, J., et al. 2014, MNRAS, 445, 2424 
V. V. Neustroev et al.: Multi-epoch tomographic study of HT Cas

Ogilvie, G. I. 2002, MNRAS, 330, 937

Oke, J. B. 1990, AJ, 99, 1621

Osaki, Y. 1989, PASJ, 41, 1005

Paczynski, B. 1977, ApJ, 216, 822

Papadaki, C., Boffin, H. M. J., Steeghs, D., \& Schmidtobreick, L. 2008, A\&A, 487,611

Patterson, J. 1981, ApJS, 45, 517

Rafanelli, P. 1979, A\&A, 76, 365

Robertson, J. W., \& Honeycutt, R. K. 1996, AJ, 112, 2248

Schmidtobreick, L., Papadaki, C., Tappert, C., \& Ederoclite, A. 2008, MNRAS, 389,1345

Schmidtobreick, L., Tappert, C., \& Saviane, I. 2003, MNRAS, 342, 145

Schneider, D. P., \& Young, P. 1980, ApJ, 238, 946

Shafter, A. W. 1983, ApJ, 267, 222

Shafter, A. W., \& Szkody, P. 1984, ApJ, 276, 305

Shafter, A. W., Szkody, P., \& Thorstensen, J. R. 1986, ApJ, 308, 765

Shakura, N. I., \& Sunyaev, R. A. 1973, A\&A, 24, 337

Skidmore, W., Mason, E., Howell, S. B., et al. 2000, MNRAS, 318, 429

Smak, J. 1969, Acta Astron., 19, 155

Smak, J. 1970, Acta Astron., 20, 311

Smak, J. 1981, Acta Astron., 31, 395

Smith, A. J., Haswell, C. A., \& Hynes, R. I. 2006, MNRAS, 369, 1537

Spruit, H. C. 1998, ArXiv e-prints [arXiv: astro-ph/9806141]
Spruit, H. C., \& Rutten, R. G. M. 1998, MNRAS, 299, 768

Steeghs, D., \& Stehle, R. 1999, MNRAS, 307, 99

Steeghs, D., Harlaftis, E. T., \& Horne, K. 1997, MNRAS, 290, L28

Tappert, C., \& Hanuschik, R. 2001, in Astrotomography, Indirect Imaging Methods in Observational Astronomy, eds. H. M. J. Boffin, D. Steeghs, \& J. Cuypers (Berlin: Springer Verlag), Lect. Notes Phys., 573, 119

Tappert, C., Mennickent, R. E., Arenas, J., Matsumoto, K., \& Hanuschik, R. W. 2003, A\&A, 408, 651

Truss, M. R. 2007, MNRAS, 376, 89

Unda-Sanzana, E., Marsh, T. R., \& Morales-Rueda, L. 2006, MNRAS, 369, 805

Verbunt, F., \& Rappaport, S. 1988, ApJ, 332, 193

Vrielmann, S., Hessman, F. V., \& Horne, K. 2002, MNRAS, 332, 176

Warner, B. 1995, Cataclysmic variable stars, Camb. Astrophys. Ser., 28

Wenzel, W. 1987, Astron. Nachr., 308, 75

Williams, G. 1983, ApJS, 53, 523

Wood, J. H., Horne, K., \& Vennes, S. 1992, ApJ, 385, 294

Wood, J. H., Naylor, T., Hassall, B. J. M., \& Ramseyer, T. F. 1995, MNRAS, 273, 772

Yakin, D. G., Suleimanov, V. F., Borisov, N. V., Shimanskii, V. V., \& Bikmaev, I. F. 2011, Astron. Lett., 37, 845

Young, P., Schneider, D. P., \& Shectman, S. A. 1981, ApJ, 245, 1035

Zhang, E.-H., Robinson, E. L., \& Nather, R. E. 1986, ApJ, 305, 740

Zharikov, S., Tovmassian, G., Aviles, A., et al. 2013, A\&A, 549, A77 